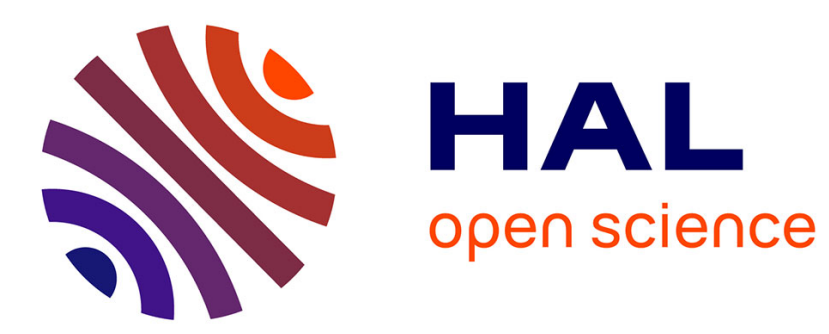

\title{
Effective thermal conductivity of periodic composites with highly conducting imperfect interfaces
}

\author{
H. Le Quang, T.-L. Phan, Guy Bonnet
}

\section{To cite this version:}

H. Le Quang, T.-L. Phan, Guy Bonnet. Effective thermal conductivity of periodic composites with highly conducting imperfect interfaces. International Journal of Thermal Sciences, 2011, 50 (8), pp.1428-1444. 10.1016/j.ijthermalsci.2011.03.009 . hal-00692837

\section{HAL Id: hal-00692837 \\ https://hal.science/hal-00692837}

Submitted on 6 Mar 2016

HAL is a multi-disciplinary open access archive for the deposit and dissemination of scientific research documents, whether they are published or not. The documents may come from teaching and research institutions in France or abroad, or from public or private research centers.
L'archive ouverte pluridisciplinaire HAL, est destinée au dépôt et à la diffusion de documents scientifiques de niveau recherche, publiés ou non, émanant des établissements d'enseignement et de recherche français ou étrangers, des laboratoires publics ou privés. 


\title{
Effective thermal conductivity of periodic composites with highly conducting imperfect interfaces
}

\author{
H. Le Quang*, T.-L. Phan, G. Bonnet
}

Université Paris-Est, Laboratoire Modélisation et Simulation Multi Echelle, MSME UMR 8208 CNRS, 5 Boulevard Descartes, F-77454 Marne-la-Vallée Cedex 2, France

\section{A B S T R A C T}

The purpose of this work is to determine the effective conductivity of periodic composites accounting for highly conducting imperfect interfaces between the matrix and inclusions phases and to study the dependencies of the effective conductivity on the size and distribution of inhomogeneities in the matrix phase in different cases: squared, hexagonal, cubic and random inclusion distributions. The local solution of the periodic conduction problem is found in Fourier space by using the Green operators and closedform expressions of factors depending on the size and shape of the inclusions. The numerical results of size-dependent effective thermal conductivity are finally compared with an analytical estimation obtained from the generalized self-consistent model. The method elaborated and results provided by the present work are directly applicable to other physically analogous transport phenomena, such as electric conduction, dielectrics, magnetism, diffusion and flow in porous media and to the mathematically identical phenomenon of anti-plane elasticity.

Keywords:

\section{Thermal properties \\ Particles-reinforced composites \\ Interface \\ Transport properties \\ Size effects}

\section{Introduction}

Recently, different works have been devoted to study the sizedependent mechanical behavior in nanosystems incorporating surface/interface energies. Indeed, when the inclusion size is diminished to the nano-scale, due to the large surface-to-volume ratio, the matrix-inclusion interface energy can no longer be neglected. This fact has been emphasized and exploited in recent investigations on nanomaterials and nano-structural elements (see, e.g. $[11,24])$. In this context, in order to estimate the size-dependent overall elastic properties of nanocomposites and nano-structural elements accounting for the surface/interface energies, the classical perfect interface is modified by adopting a coherent interface model in which the displacement vector field is continuous across an interface while the stress vector field is discontinuous across the same interface $([2,29,31])$. The thermal conduction counterpart of the interface stress and energy model is the highly conducting (HC) interface model, which is the subject of the present paper. More precisely, according to this imperfect interface model, the temperature is continuous across this interface but the normal heat flux component is discontinuous across the same interface due to the possibility of having a surface heat flux along the interface whose surface energy conservation equation gives rise to the

\footnotetext{
* Corresponding author. Tel.: +33 (0) 160957 797; fax: +33 (0) 160957799. E-mail address: hung.lequang@univ-paris-est.fr (H. Le Quang).
}

generalized Young-Laplace equation. These interface conditions for the HC interface model are completely contrary to the ones of the well-known Kapitza interface thermal resistance model which has been recognized to be of a great theoretical and practical importance in physics and materials science (see e.g. [17]). By accounting for the thermal resistance appearing at the interface between two bulk media, the Kapitza interface thermal resistance model stipulates that the temperature suffers a jump across the interface while the normal heat flux component is continuous across the same interface and usually taken to be proportional to the temperature jump. Thus, the HC interface model can be viewed as dual with respect to the Kapitza interface thermal resistance model. The physical background of both HC interface model and thermal resistance interface model can be clarified by considering the general imperfect interface model in which a very thin interphase of uniform thickness is situated between two bulk phases. By applying an asymptotic approach to this interphase to obtain appropriate temperature and normal heat flux component jump conditions for an interface of zero thickness replacing the interphase, Sanchez-Palencia [30] and Pham Huy and Sanchez-Palencia [28] showed that the general imperfect interface model reduces to the $\mathrm{HC}$ interface model or the thermal resistance interface model according as the interphase is highly conducting or slowly conducting with respect to the surrounding phases. In other words, the $\mathrm{HC}$ interface model and the thermal resistance interface model may be considered as the two limiting cases of the general imperfect interface model. 
There are several possible methodologies in deriving the sizedependent overall thermal properties of composites affected by the significant size effects appearing in conducting composites containing $\mathrm{HC}$ imperfect interfaces. These possible interests can be classified into three categories.

The first category includes all analytical estimation methods based either on the generalized Eshelby's formalism, such as diluted, self-consistent, generalized self-consistent, differential schemes and those of Mori-Tanaka or on the ellipsoidal harmonic solution for the Laplace equation. In ref. [18], the Eshelby's results and formalism for an circular or spherical inhomogeneity embedded in an elastic infinite matrix are extended to the thermal conduction phenomenon accounting for the $\mathrm{HC}$ imperfect interface between matrix and inclusions. Quite different from the relevant results of elasticity, Le Quang et al. [18] showed that the generalized Eshelby's conduction tensor fields and localization tensor fields inside circular and spherical inhomogeneities remain uniform even in the presence of the $\mathrm{HC}$ imperfect interface. Then, the analytical closed-form expressions fo thermal effective conductivity has been derived as functions of interface properties and of the inhomogeneities size. Another approach employing the ellipsoidal harmonic and analytical solution for the Laplace equation to calculate the effective thermal conduc tivity has been presented in refs. [9,25]. However, these last two works are limited to the case where the phases constituting the composite are isotropic.

The second category contains all methods based on variational principles to determine the bounds of size-dependent effective thermal properties. In refs. [6,19-21], by using the generalized Hashin-Shtrikman variational principles and by constructing appropriate trial fields, the explicit upper and lower bounds are obtained for the effective thermal conductivity of composite materials consisting of two anisotropic phases. Recently, these results have been generalized by Lipton and Talbot [22] aiming at finding the upper and lower bounds for the effective therma conductivity of composite with a nonlinear imperfect interface.

The third category associated to the present study includes all the numerical methods. Previous methods use mainly the finite element method with surface elements or the level set method to describe the HC imperfect interface (e.g. [35]), while the method used in the present paper is based on the fast Fourier transform (FFT) of the solution. The method based on FFT has been used frequently to compute the effective properties of periodic composites in the context of linear or nonlinear elasticity (e.g. [3,23,27]) and has been generalized recently to the context of piezoelectricity by Brenner [5] However, the results presented in refs. [3,5,23,27] were limited to the case where the interfaces between matrix and inclusion phases are perfect.

The present work is concerned with the thermal conduction phenomenon which plays an important area in the fields of materials science and solid-state physics. The elaborated method and the results obtained for heat conduction are directly applicable to othe transport phenomena like electric conduction, dielectrics, magnetism, diffusion and flow in porous media and to anti-plane elasticity problem. This is due to the fact that the thermal conduction problem is physically analogous to other problems involving transport phenomena such as electric conduction, dielectrics, magnetism, diffusion and flow in porous media and that there is a correspondence between 2D conduction and anti-plane elasticity (see, e.g. [26]). The present work has two objectives. First, it aims at extending the alternate method based on Fourier series $([3,23,27])$ in the context of linear elasticity to the thermal conduction phenomenon including the effect of the highly conducting imperfect interface. The generalized method proposed uses the FFT and an iterative process to solve the local solution of periodic conduction problem in the three following cases: with squarely, hexagonally and randomly distributed inclusions. Second, it has the purpose of employing the solution of the localization problem to determine and study the effects of the interface, size and distribution of inclusions on the effective thermal properties of periodic composites.

The paper is organized as follows. Section 2 is dedicated to specifying the constitutive laws of the constituent phases of composites under investigation, the $\mathrm{HC}$ interface model and the general form of the effective thermal behavior. In Section 3 , the solution of the localization periodic problem is presented in the Fourier space in the context of conduction phenomenon with $\mathrm{HC}$ imperfect interface. Then, the size-dependent effective conductivities are calculated from the local solution. In Section 4, the HC interface and inhomogeneities size effects as well as the dependence of the distribution of inhomogeneities in the matrix phase on the effective conductivity of periodic composites are numerically discussed and illustrated; in addition, the numerically obtained results are compared with the results provided by using the estimation with the help of the generalized self-consistent mode (GSCM) and matrix/interphase/inclusion configuration. In Section 5 , a few conclusions are given.

\section{Local constitutive laws}

In this section, we specify the local constitutive laws of the twodimensional (2D) or three-dimensional (3D) periodic composite studied in this work. Let $\Omega$ be a representative volume element (RVE) consisting of a host matrix medium in which $M(\geq 1)$ inclusions are embedded. The matrix, referred to phase 2 and occupied by $\Omega^{(m)}$ and inclusions, denoted as phase 1 , are assumed to be individually homogeneous and have the linear thermal behavior described by Fourier's law

\section{$\mathbf{q}(\mathbf{x})=\mathbf{K}(\mathbf{x}) \varepsilon(\mathbf{x})$}

Here $\varepsilon(\mathbf{x})$ being the intensity field is related to the temperature field $T(\mathbf{x})$ by

$\varepsilon(\mathbf{x})=-\nabla T(\mathbf{x})$

The local thermal conductivity tensor $\mathbf{K}(\mathbf{x})$ is written as

$\mathbf{K}(\mathbf{x})=\sum_{i=1}^{M} \chi^{(i)}(\mathbf{x}) \mathbf{K}^{(1)}+\left[1-\sum_{i=1}^{M} \chi^{(i)}(\mathbf{x})\right] \mathbf{K}^{(2)}$,

where $\mathbf{K}^{(\alpha)}$, with $\alpha=1$ or 2 , stands for the thermal conductivity tensor of phase $\alpha$, and $\chi^{(i)}(\mathbf{x})$ is the characteristic function of the $i$ thinclusion $(M \geq i \geq 1)$, occupied by $\Omega^{(i)}$, such as $\chi^{(i)}(\mathbf{x})=1$ for $\mathbf{x} \in \Omega^{(i)}$ and $\chi^{(i)}(\mathbf{x})=0$ for $\mathbf{x} \notin \Omega^{(i)}$. The heat field $\mathbf{q}(\mathbf{x})$ complies with the energy conservation equation which is written as

$\nabla \cdot \mathbf{q}(\mathbf{x})=0$

in the case of the stationary thermal conduction without heat source.

The interface between the matrix and the ith-inclusion, denoted by $\Gamma^{(i)}$, is modeled by a coherent interface model. This model was first proposed by refs. $[7,12,31]$ in the context of elasticity, then extended to thermal conduction phenomenon by ref. [8]. According to this model for thermal conduction phenomenon, the matrix-inclusion interface is considered as a conducting body $\Gamma^{(i)}$ with a vanishing thickness across which the temperature field $T(\mathbf{x})$ is continuous. Indeed, it follows from Hadamard's relation (see, e.g. [16]) that the tangential projection $\varepsilon^{s}(\mathbf{x})$ of the intensity field $\varepsilon(\mathbf{x})$ is continuous even though the intensity field $\varepsilon(\mathbf{x})$ is generally discontinuous across $\Gamma^{(i)}$. By introducing the tangential projection operator $\mathbf{P}$ defined as 


$$
\mathbf{P}(\mathbf{x})=\mathbf{I}^{(d)}-\mathbf{n}(\mathbf{x}) \otimes \mathbf{n}(\mathbf{x}),
$$

with $d=2$ or 3 according to the cases 2D or 3D, respectively, $\mathbf{I}^{(d)}$ standing for the $d$-dimensional second-order identity tensor and $\mathbf{n}(\mathbf{x})$ denoting the outward normal to $\Gamma^{(i)}$, the surface intensity field $\varepsilon^{s}(\mathbf{x})$ on $\Gamma^{(i)}$ is then expressed as

$\varepsilon^{S}(\mathbf{x})=\mathbf{P}(\mathbf{x}) \varepsilon(\mathbf{x})$.

Within the matrix-inclusion interface, the surface heat flux field $\mathbf{q}^{s}(\mathbf{x})$ is related to the surface intensity field $\varepsilon^{s}(\mathbf{x})$ by the following thermal linear isotropic behavior

$\mathbf{q}^{s}(\mathbf{x})=k_{s}^{(i)} \varepsilon^{s}(\mathbf{x})$,

where $\mathbf{x} \in \Gamma^{(i)}$ and $k_{s}^{(i)}$ is the surface thermal conductivity of $\Gamma^{(i)}$ Unlike the classical case where the interface is perfect, the normal component of the heat flux field $\mathbf{q}(\mathbf{x})$ is in general discontinuous across the coherent interface $\Gamma^{(i)}$ and its jump is related to the surface heat flux field $\mathbf{q}^{s}(\mathbf{x})$ by the following surface energy conservation equation

$$
\left(\mathbf{q}^{(2)}-\mathbf{q}^{(1)}\right) \cdot \mathbf{n}(\mathbf{x})=-\nabla_{s} \cdot \mathbf{q}^{(s)}(\mathbf{x}), \quad \mathbf{x} \in \Gamma^{(i)},
$$

where $\nabla_{S} \cdot \mathbf{q}^{S}(\mathbf{x})$ represents the surface divergence of the surface heat flux field $\mathbf{q}^{s}(\mathbf{x})$. In particular, $\nabla_{s} \cdot \mathbf{q}^{s}(\mathbf{x})$ takes the form

$\nabla_{S} \cdot \mathbf{q}^{S}(\mathbf{x})=\frac{\partial q_{\theta}^{S}}{r \partial \theta}+\frac{q_{\theta}^{s}}{r} \cot \theta+\frac{1}{r \sin \theta} \frac{\partial q_{\varphi}^{S}}{\partial \varphi}$

in the spherical coordinate system $(r, \theta, \phi)$ or

$\nabla_{S} \cdot \mathbf{q}^{S}(\mathbf{x})=\frac{\partial q_{\theta}^{S}}{r \partial \theta}$

in the polar coordinate system $(r, \theta)$

Next, in order to clarify the physical background and the validity domain of the coherent interface model used to describe $\Gamma^{(i)}$, we consider in Fig. 1 two configurations. In the three-phase one, the representative volume element $\Omega$ consists of the inclusion phase $\Omega^{(i)}$ embedded in to the matrix phase $\Omega^{(m)}$ via the interphase $\Omega^{(c i)}$ (see e.g. $[1,4,14]$ ). According to the three-phase model, the interface located between $\Omega^{(i)}$ and $\Omega^{(c i)}$ and the one between $\Omega^{(m)}$ and $\Omega^{(c i}$ are assumed to be perfect, and the thickness $h^{(i)}$ of $\Omega^{(c i)}$ is required to be uniform and small in comparison with the inclusion size. In the second configuration, the interphase is now replaced by an interphase of zero thickness $\Gamma^{(i)}$, namely an imperfect interface. By using an asymptotic expansion, the jump conditions that the imperfect interface $\Gamma^{(i)}$ has to verify for the two configurations to be physically equivalent were derived by refs. $[28,30]$. More precisely, when the interphase with thermal conductivity tensor $\mathbf{K}_{c}^{(i)}$ is assumed to be highly conducting, or equivalently $\left\|\mathbf{K}_{c}^{(i)}\right\|>>\left\|\mathbf{K}^{(1)}\right\|$ and $\left\|\mathbf{K}_{c}^{(i)}\right\|>>\left\|\mathbf{K}^{(2)}\right\|$, then the conditions that the imperfect interface $\Gamma^{(i)}$ must satisfy are those of the coherent interface model described above. The corresponding surface thermal conductivity tensor $\mathbf{K}_{s}^{(i)}$ of $\Gamma^{(i)}$ can be expressed in terms of $\mathbf{K}_{c}^{(i)}$ and $h^{(i)}$ as follows (see $[28,30])$ :

$\mathbf{K}_{\mathrm{s}}^{(i)}=h^{(i)} \mathbf{S}_{c}^{(i)}$

where

$\mathbf{S}_{c}^{(i)}=\mathbf{K}_{c}^{(i)}-\frac{\left(\mathbf{K}_{c}^{(i)} \mathbf{n}\right) \otimes\left(\mathbf{K}_{c}^{(i)} \mathbf{n}\right)}{\mathbf{K}_{c}^{(i)}:(\mathbf{n} \otimes \mathbf{n})}$.

In the present work, $\mathbf{K}_{c}^{(i)}$ is assumed to be isotropic, i.e. $\mathbf{K}_{c}^{(i)}=k_{c}^{(i)} \mathbf{I}^{(d)}$ with $k_{c}^{(i)}$ standing for the thermal conductivity of the interphase and it is immediate from Eqs. (11) and (12) that

$\mathbf{K}_{\mathrm{s}}^{(i)}=k_{\mathrm{s}}^{(i)} \mathbf{P}, \quad k_{\mathrm{s}}^{(i)}=h^{(i)} k_{c}^{(i)}$.

At the macroscopic scale, the composite under consideration is assumed to be homogeneous. The corresponding effective thermal behavior is written as

$\mathbf{Q}=\mathbf{K}^{\text {eff }} \mathbf{E}$,

where $\mathbf{K}^{\text {eff }}$ is the effective thermal conductivity second-order tensor and the macroscopic heat flux $\mathbf{Q}$ and intensity fields $\mathbf{E}$ over a representative volume element are defined by

$\mathbf{Q}=\frac{1}{|\Omega|} \int_{\partial Q}(\mathbf{q} \cdot \boldsymbol{v}) \mathbf{x} \mathrm{d} \mathbf{x}, \quad \mathbf{E}=-\frac{1}{|\Omega|} \int_{\partial \Omega} T(\mathbf{x}) \boldsymbol{v}(\mathbf{x}) \mathrm{d} \mathbf{x}$,

where $\boldsymbol{\nu}(\mathbf{x})$ is the outward unit normal vector to $\partial \Omega$ and $|\Omega|$ denotes the volume or surface of $\Omega$ according as the 3D or 2D case is concerned.
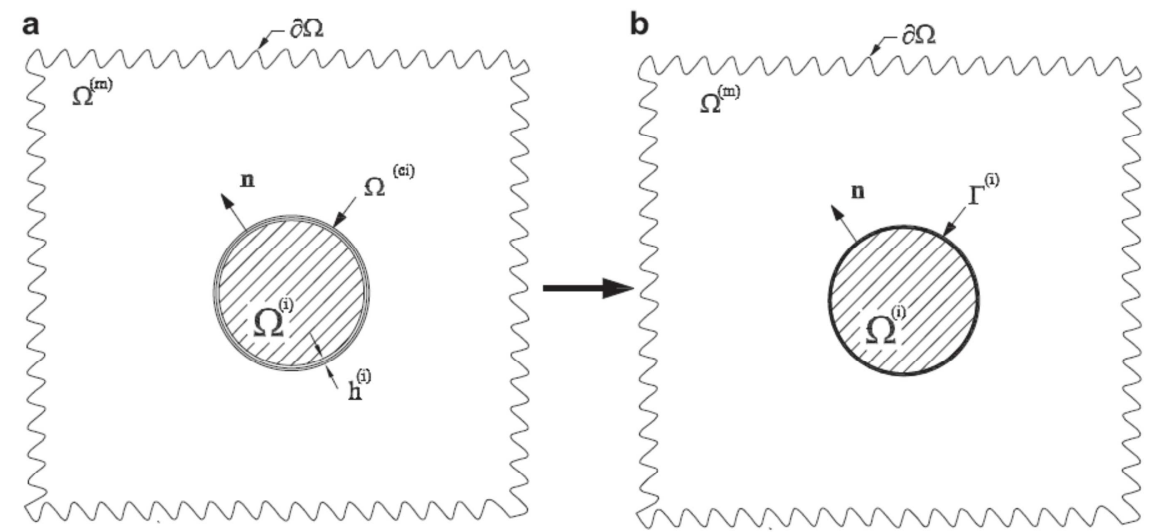

Fig. 1. Two equivalent configurations: (a) three-phase matrix/interphase/inclusion periodic composite; (b) two-phase matrix/inclusion periodic composite with imperfect transmission conditions. 
As in the classical case with perfect interface, the macroscopic intensity field $\mathbf{E}$ can be determined by the volume or surface average of its local counterpart over a representative volume element. However, due to the discontinuity of the normal heat flux field component across the coherent interface, the macroscopic heat flux field $\mathbf{Q}$ is not simply the volume or surface average of the local counterpart over a representative volume element and it is given by

$\mathbf{Q}=c_{1} \overline{\mathbf{q}}^{(1)}+c_{2} \overline{\mathbf{q}}^{(2)}+\frac{1}{|\Omega|} \sum_{j=1}^{M} \int_{\Gamma^{(i)}}\left(\mathbf{q}^{(2)} \cdot \mathbf{n}-\mathbf{q}^{(1)} \cdot \mathbf{n}\right) \mathbf{x} \mathrm{d} \mathbf{x}$,

or equivalently

$\mathbf{Q}=c_{1} \overline{\mathbf{q}}^{(1)}+c_{2} \overline{\mathbf{q}}^{(2)}-\frac{1}{|\Omega|} \sum_{j=1}^{M} \int_{\Gamma^{(i)}}\left(\nabla_{S} \cdot \mathbf{q}^{S}\right) \mathbf{x} \mathrm{d} \mathbf{x}$,

where $c_{\alpha}$ is the volume fraction of phase $\alpha$ and $\overline{\mathbf{q}}^{(\alpha)}$ denotes the volume or surface average in phase $\alpha$ of the heat flux field $\mathbf{q}(\mathbf{x})$.

\section{Solution of the localization problem}

First, let $\Omega$ be subjected to the following uniform intensity field boundary condition

$T(\mathbf{x})=-\mathbf{E} \cdot \mathbf{x}, \quad \mathbf{x} \in \partial \Omega$

where $\mathbf{E}$ is a constant intensity vector. Under the boundary condition Eq. (18), as for the classical case with perfect interfaces, owing to the fact that the temperature field $T(\mathbf{x})$ is continuous across the coherent imperfect interfaces $\Gamma^{(i)}$, it is immediate from Eq.(15) that the macroscopic intensity field is equal to $\mathbf{E}$.

In order to calculate the local intensity and heat flux fields in $\Omega$, we consider now a typical parallelepiped unit cell $U$ which is defined by

$U=\left\{\mathbf{x} \in \Omega \mid-\lambda_{m} \leq x_{m} \leq \lambda_{m}\right\}$

with $m=1,2$ for the 2D case or $m=1,2,3$ for the 3D case. Different unit cells of some typical periodic composite are studied in detail in this work (Fig. 2).

Next, by introducing a "reference medium" whose thermal conductivity is denoted by $\mathbf{K}^{0}$ and by accounting for the discontinuity of the heat flux field $\mathbf{q}(\mathbf{x})$ across interface $\Gamma$, the energy conservation equation Eq. (4) becomes

$\nabla \cdot \mathbf{q}(\mathbf{x})=\nabla \cdot\left[\left(\mathbf{K}^{0}+\Delta \mathbf{K}\right) \varepsilon(\mathbf{x})\right]-\sum_{i=1}^{N}\left(\mathbf{q}^{(2)}-\mathbf{q}^{(1)}\right) \cdot \mathbf{n} \delta_{\Gamma^{(i)}}(\mathbf{x})=0$,

where $N$ denotes the number of inclusions embedded in a unit cell and $\delta_{\Gamma^{(i)}}(\mathbf{x})$ is the Dirac delta function over the interface $\Gamma^{(i)}$ between the matrix and the ith-inclusion and

$\Delta \mathbf{K}(\mathbf{x})=\mathbf{K}(\mathbf{x})-\mathbf{K}^{0}$.

In addition, an inclusion is called embedded in the unit cell $U$ if the position of its center is inside $U$.

To perform the homogenization process, the intensity field $\varepsilon(\mathbf{x})$ is split into the uniform and periodic perturbation parts, $\mathbf{E}$ and $\varepsilon^{*}(\mathbf{x})$, such that $\varepsilon(\mathbf{x})=\mathbf{E}+\varepsilon^{*}(\mathbf{x})$. The temperature field related to the periodic intensity filed $\varepsilon^{*}(\mathbf{x})$ is denoted by $T^{*}(\mathbf{x})$. Moreover, when the $i$ th-inclusion is $2 \mathrm{D}$ circular or 3D spherical of radius $R_{i}$, it is shown in Appendix A that the following identity holds on the interface $\Gamma^{(i)}$ between the matrix and ith-inclusion $\nabla_{s} \cdot(\mathbf{P v})=-\frac{(d-1)}{R_{i}} \mathbf{v} \cdot \mathbf{n}+\nabla_{s} \cdot \mathbf{v}$,

for any continuously differentiable vector v. By combining Eqs. (6)-(8) with Eq. (20) and by accounting for Eq. (22), Eq. (20) can be rewritten in the equivalent form

$$
\begin{gathered}
-\nabla \cdot\left[\mathbf{K}^{0} \nabla T^{*}(\mathbf{x})\right]+\nabla \cdot \tau(\mathbf{x})+\sum_{i=1}^{N} k_{s}^{(i)} \nabla_{S} \cdot \varepsilon(\mathbf{x}) \delta_{T^{(i)}}(\mathbf{x}) \\
-\sum_{i=1}^{N} \frac{(d-1) k_{S}^{(i)}}{R_{i}} \varepsilon(\mathbf{x}) \cdot \mathbf{n}(\mathbf{x}) \delta_{T^{(i)}}(\mathbf{x})=0
\end{gathered}
$$

where the "polarization field" $\tau(\mathbf{x})$ is defined as

$\tau(\mathbf{x})=\Delta \mathbf{K}(\mathbf{x})\left[\mathbf{E}+\varepsilon^{*}(\mathbf{x})\right]$

The temperature, intensity and polarization fields can be written as follows

$T^{*}(\mathbf{x})=\sum_{\xi}^{N_{k}} \widehat{T}^{*}(\xi) e^{l \xi \cdot \mathbf{x}}, \quad \tau(\mathbf{x})=\sum_{\xi}^{N_{k}} \widehat{\tau}(\xi) e^{\ell \cdot \mathbf{x}}$,

$\varepsilon(\mathbf{x})=\sum_{\xi^{\prime}}^{N_{k}} \widehat{\varepsilon}\left(\xi^{\prime}\right) e^{l \xi^{\prime} \cdot \mathbf{x}}$,

where $\iota=\sqrt{-1}$, and $\widehat{T}^{*}(\xi), \widehat{\varepsilon}\left(\xi^{\prime}\right)$ and $\widehat{\tau}(\xi)$ stand for the discrete Fourier transforms of $T^{*}(\mathbf{x}), \varepsilon(\mathbf{x})$ and $\tau(\mathbf{x})$, respectively; $\xi$ and $\xi^{\prime}$ are the discrete wave vectors arranged along a discrete network having a period $\pi / \lambda_{m}$ following the direction $m ; N_{k}$ is the number of discrete wave vectors. Substituting Eq. (25) into Eq. (23) yields

$$
\begin{aligned}
\sum_{\xi}^{N_{k}}\left(\xi_{m} K_{m j}^{0} \xi_{j}\right) \widehat{T}^{*}(\xi) e^{i \xi \cdot \mathbf{x}}+\sum_{\xi}^{N_{k}} i \xi_{m} \widehat{\tau}_{m}(\xi) e^{\imath \xi \cdot \mathbf{x}}+\sum_{\xi^{\prime}}^{N_{k}} \widehat{\varepsilon}_{m}\left(\xi^{\prime}\right) h_{m} \\
-\sum_{\xi^{\prime}}^{N_{k}} \hat{\varepsilon}_{m}\left(\xi^{\prime}\right) p_{m}=0,
\end{aligned}
$$

where

$h_{m}=\sum_{i=1}^{N} k_{s}^{(i)} \delta_{\Gamma^{(i)}}(\mathbf{x}) \nabla_{S} \cdot\left(e^{\left.i \xi^{\prime} \cdot \mathbf{x}_{\mathbf{f}_{m}}\right)}\right.$,

$p_{m}=(d-1) \sum_{i=1}^{N} \frac{k_{s}^{(i)}}{R_{i}} \delta_{T^{(i)}}(\mathbf{x}) n_{m} e^{l \xi^{\prime} \cdot \mathbf{x}}$,

with $\mathbf{f}_{m}$ standing for the basis unit vector following the direction $m$ in a Cartesian coordinate system $\left\{x_{1}, x_{2}, x_{3}\right\}$. Correspondingly, the Fourier transform components of the distributions $h_{m}$ and $p_{m}$ are given by:

$\widehat{h}_{m}\left(\xi, \xi^{\prime}\right)=\frac{1}{|U|} \sum_{i=1}^{N} k_{s}^{(i)} \int_{\Gamma^{(i)}} \nabla_{s} \cdot\left(e^{i \xi^{\prime} \cdot \mathbf{x}} \mathbf{f}_{m}\right) e^{-i \xi \cdot \mathbf{x}} \mathrm{d} \mathbf{x}$,

$\widehat{p}_{m}\left(\xi, \xi^{\prime}\right)=\frac{d-1}{|U|} \sum_{i=1}^{N} \frac{k_{s}^{(i)}}{R_{i}} \int_{\Gamma^{(i)}} n_{m}(\mathbf{x}) e^{-i\left(\xi-\xi^{\prime}\right) \cdot \mathbf{x}} \mathrm{d} \mathbf{x}$.

Here, the surface divergence on $\Gamma^{(i)}$ of an arbitrary differentiable vector $\mathbf{v}$ is defined as

$\nabla_{S} \cdot \mathbf{v}=\operatorname{Tr}(\nabla \mathbf{v P})$

where $\operatorname{Tr}(\bullet)$ denotes the trace of the tensor $\bullet$ and $\mathbf{P}$ is defined by Eq. (5). By setting $\mathbf{x}=\tilde{\mathbf{x}}+\mathbf{X}^{(i)}$ with $\mathbf{x} \in \Gamma^{(i)}$ and $\mathbf{X}^{(i)}$ being the 


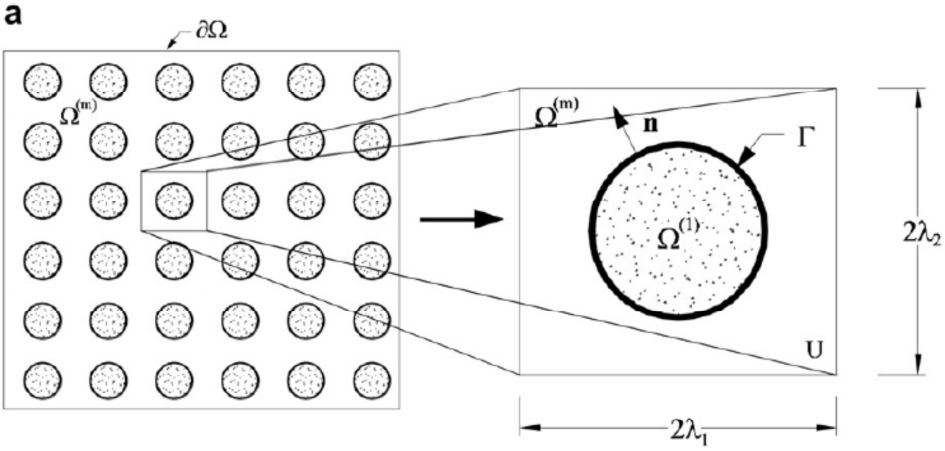

b
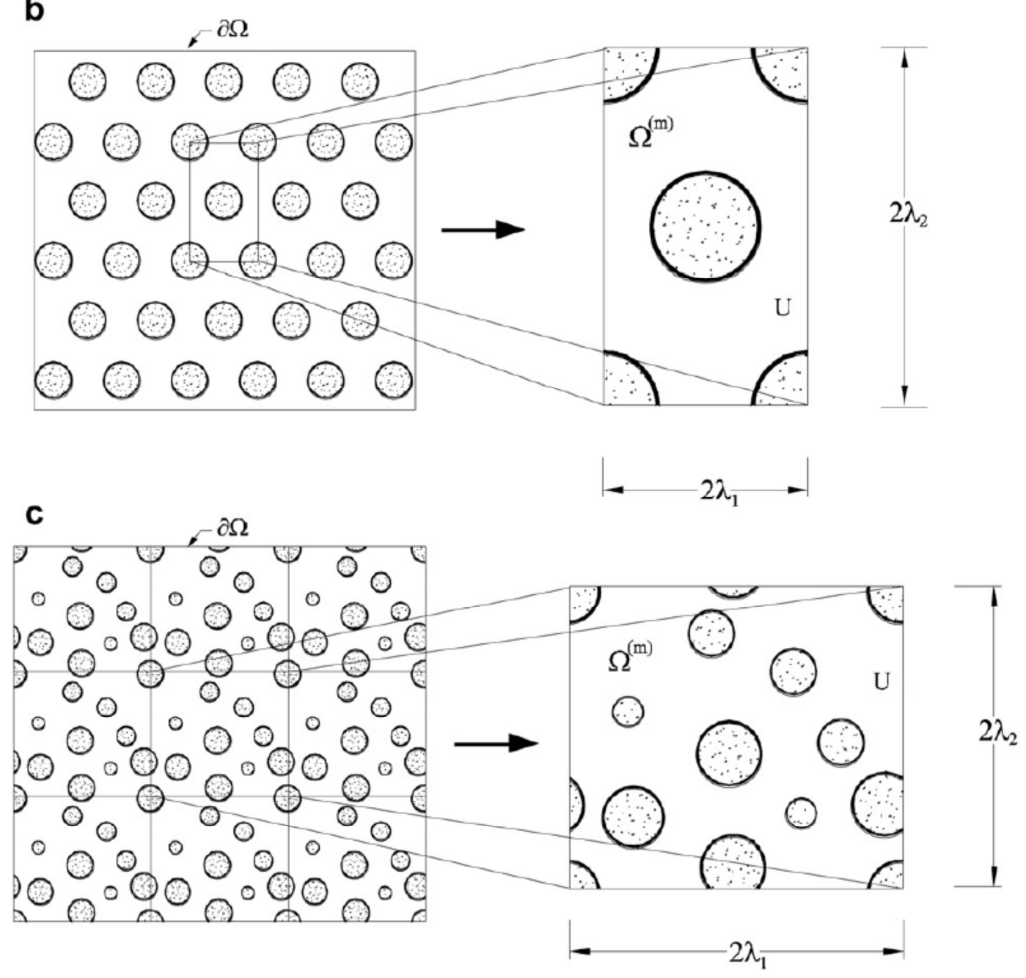

Fig. 2. (a) Representative volume element (RVE) and typical unit cell $U$ of a periodic composite with squarely distributed inclusions; (b) RVE and typical unit cell $U$ of a periodic composite with hexagonally distributed inclusions; (c) RVE and typical unit cell $U$ of a periodic composite with 10 randomly distributed inclusions.

position vector of the center of $i$ th-inclusion, the computation of $h_{m}\left(\xi, \xi^{\prime}\right)$ and $\widehat{p}_{m}\left(\xi, \xi^{\prime}\right)$ when the ith-inclusion is circular or spherical of radius $R_{i}$ leads to

$$
\begin{aligned}
& \widehat{h}_{m}\left(\xi, \xi^{\prime}\right)=\frac{1}{|U|} \sum_{i=1}^{N} k_{s}^{(i)} \widehat{H}_{m l}^{(i)}\left(\xi-\xi^{\prime}\right) \xi_{l}^{\prime}, \\
& \widehat{p}_{m}\left(\xi, \xi^{\prime}\right)=\frac{d-1}{|U|} \sum_{i=1}^{N} k_{s}^{(i)} \widehat{P}_{m}^{(i)}\left(\xi-\xi^{\prime}\right),
\end{aligned}
$$

where $\widehat{H}_{m l}^{(i)}$ is the component of the $2 \times 2$ or $3 \times 3$ matrix $\widehat{\mathbf{H}}^{(i)}\left(\xi-\xi^{\prime}\right.$ according respectively to the $2 \mathrm{D}$ circular or $3 \mathrm{D}$ spherical inclusion case; $\widehat{P}_{m}^{(i)}$ is the component of the 2D or 3D vector $\widehat{\mathbf{P}}^{(i)}\left(\boldsymbol{\xi}-\boldsymbol{\xi}^{\prime}\right)$. The expressions for $\widehat{H}_{m l}^{(i)}$ and $\widehat{P}_{m}^{(i)}$ are detailed in Appendix $C$. Thus, we have

$$
\begin{aligned}
\sum_{\xi^{\prime}}^{N_{k}} \widehat{\mathbf{h}}\left(\xi, \xi^{\prime}\right) \cdot \widehat{\varepsilon}\left(\xi^{\prime}\right) & =\frac{1}{|U|} \sum_{i=1}^{N} k_{s}^{(i)}\left[\widehat{H}_{m l}^{(i)} * \widehat{\epsilon}_{l m}\right](\xi), \\
\sum_{\xi^{\prime}}^{N_{k}} \widehat{\mathbf{p}}\left(\xi, \xi^{\prime}\right) \cdot \widehat{\varepsilon}\left(\xi^{\prime}\right) & =\frac{d-1}{|U|} \sum_{i=1}^{N} k_{s}^{(i)}\left[\widehat{P}_{m}^{(i)} * \widehat{\varepsilon}_{m}\right](\xi),
\end{aligned}
$$

where the symbol * denotes the convolution product and the Fourier component $\widehat{\epsilon}_{m l}$ is defined as 


$$
\widehat{\epsilon}_{m l}\left(\xi^{\prime}\right)=\xi_{l}^{\prime} \widehat{\varepsilon}_{m}\left(\xi^{\prime}\right) \text {. }
$$

Therefore, Eq. (26) can be expressed in terms of Fourier transforms as

$$
\begin{aligned}
\widehat{T}^{*}(\xi)= & -\frac{\iota \xi}{\xi \cdot \mathbf{K}^{0} \xi} \cdot \hat{\tau}(\xi)-\frac{1}{|U| \xi \cdot \mathbf{K}^{0} \xi} \sum_{i=1}^{N} k_{s}^{(i)}\left(\widehat{\mathbf{H}}^{(i)} * \hat{\epsilon}\right)(\xi) \\
& +\frac{(d-1)}{|U| \xi \cdot \mathbf{K}^{0} \xi} \sum_{i=1}^{N} k_{s}^{(i)}\left[\widehat{\mathbf{P}}^{(i)} * \hat{\varepsilon}\right](\xi) .
\end{aligned}
$$

Additionally, owing to the fact that $\widehat{\varepsilon}(\boldsymbol{\xi})-\widehat{\mathbf{E}}(\boldsymbol{\xi})=\widehat{\boldsymbol{\varepsilon}}^{*}(\boldsymbol{\xi})=-\iota \xi \widehat{T}^{*}(\boldsymbol{\xi})$ with

$$
\widehat{\mathbf{E}}(\boldsymbol{\xi})= \begin{cases}\mathbf{E} & \text { for } \xi=\mathbf{0}, \\ \mathbf{0} & \text { for } \xi \neq \mathbf{0}\end{cases}
$$

it follows from Eq. (34) that

$$
\begin{aligned}
\widehat{\varepsilon}(\boldsymbol{\xi})= & \widehat{\mathbf{E}}(\boldsymbol{\xi})+\widehat{\boldsymbol{I}}^{0}(\boldsymbol{\xi}) \hat{\tau}(\boldsymbol{\xi})+\frac{1}{|U|} \hat{\boldsymbol{\Lambda}}^{0}(\boldsymbol{\xi}) \sum_{i=1}^{N} k_{s}^{(i)}\left[\widehat{\mathbf{H}}^{(i)} * \hat{\epsilon}\right](\boldsymbol{\xi}) \\
& -\frac{(d-1)}{|U|} \hat{\boldsymbol{\Lambda}}^{0}(\boldsymbol{\xi}) \sum_{i=1}^{N} k_{s}^{(i)}\left[\widehat{\mathbf{P}}^{(i)} * \hat{\varepsilon}\right](\xi) .
\end{aligned}
$$

Here, the Fourier transforms of Green operators, $\widehat{\Gamma}^{0}$ and $\hat{\boldsymbol{\Lambda}}^{0}$, are respectively given by

$\widehat{T}^{0}(\xi)=-\frac{\xi \otimes \xi}{\xi \cdot \mathbf{K}^{0} \xi}, \quad \hat{\Lambda}^{0}(\xi)=\frac{\iota}{\xi \cdot \mathbf{K}^{0} \xi}$.

With the help of Eq. (24), the corresponding Fourier transforms $\widehat{\tau}(\boldsymbol{\xi})$ of the polarization field $\tau(\mathbf{x})$ is calculated by

$\widehat{\tau}(\xi)=\left(\mathbf{K}^{(2)}-\mathbf{K}^{0}\right) \hat{\varepsilon}(\boldsymbol{\xi})+\left(\mathbf{K}^{(1)}-\mathbf{K}^{(2)}\right) \sum_{i=1}^{N}\left[\hat{\chi}^{(i)} * \widehat{\varepsilon}\right](\xi)$,

where the Fourier transform $\hat{\chi}^{(i)}\left(\xi-\xi^{\prime}\right)$ of $\chi^{(i)}(\mathbf{x})$, called also shape coefficient, is given by

$\hat{\chi}^{(i)}\left(\xi-\xi^{\prime}\right)=\frac{e^{-\imath\left(\xi-\xi^{\prime}\right) \cdot \mathbf{X}^{(i)}}}{|U|} \int_{\Omega^{(i)}} e^{-\imath\left(\xi-\xi^{\prime}\right) \cdot \tilde{\mathbf{x}}} \mathrm{d} \tilde{\mathbf{x}}$.

Notice that $\hat{\chi}^{(i)}\left(\xi-\xi^{\prime}\right)$ depends on both size and shape of inclusion. In particular, $\hat{\chi}(\boldsymbol{l})\left(\xi-\xi^{\prime}\right)$ can be expressed explicitly as:

(i) when the $i$ th-inclusion is elliptic with two principal radii $a_{1}$ and $a_{2}$

$\hat{\chi}^{(i)}\left(\xi-\xi^{\prime}\right)= \begin{cases}\frac{2 S^{(i)} J_{1}(z)}{|U| z} e^{-l\left(\xi-\xi^{\prime}\right) \cdot \mathbf{X}^{(i)}} & \text { for } z \neq 0 ; \\ \frac{S^{(i)}}{|U|} e^{-\iota\left(\xi-\xi^{\prime}\right) \cdot \mathbf{X}^{(i)}} & \text { for } z=0 ;\end{cases}$

where $J_{1}$ is the Bessel function of first kind and first order; $S^{(i)}$ is the surface area of the ith-inclusion and $z=\left[\left(\xi_{1}-\xi_{1}{ }_{1}\right)^{2} a_{1}{ }^{2}+\right.$ $\left.\left(\xi_{2}-\xi_{2}^{\prime}\right)^{2} a_{2}{ }^{2}\right]^{1 / 2}$ with $\xi_{1}$ and $\xi_{2}$ (or $\xi_{1}^{\prime}{ }_{1}$ and $\xi_{2}^{\prime}$ ) being the components of $\xi$ (or $\xi^{\prime}$ ) in the direction of the axes of the ellipse.

(ii) when the $i$ th-inclusion is ellipsoidal with three principal radii $a_{1}, a_{2}$ and $a_{3}$

$\hat{\chi}^{(i)}\left(\xi-\xi^{\prime}\right)= \begin{cases}\frac{3 V^{(i)}[\sin (z)-z \cos (z)]}{|U| z^{3}} e^{-i\left(\xi-\xi^{\prime}\right) \cdot x^{(i)}} & \text { for } z \neq 0 \\ \frac{V^{(i)}}{|U|} e^{-i\left(\xi-\xi^{\prime}\right) \cdot \mathbf{x}^{(i)}} & \text { for } z=0\end{cases}$ where $V^{(i)}$ is the volume of the $i$ th-inclusion and $z=\left[\left(\xi_{1}-\right.\right.$ $\left.\left.\xi_{1}^{\prime}\right)^{2} a_{1}^{2}+\left(\xi_{2}-\xi_{2}^{\prime}\right)^{2} a_{2}{ }^{2}+\left(\xi_{3}-\xi_{3}^{\prime}\right)^{2} a_{3}{ }^{2}\right]^{1 / 2}$ with $\xi_{1}, \xi_{2}$ and $\xi_{3}\left(\xi_{1}^{\prime}{ }_{1}\right.$, $\xi^{\prime}{ }_{2}$ and $\xi_{3}^{\prime}$ ) being the components of $\xi\left(\xi^{\prime}\right)$ in the direction of the axes of the ellipsoidal inclusion.

Introducing Eq. (37) into Eq. (35) leads to

$$
\begin{aligned}
\widehat{\varepsilon}(\xi)= & \widehat{\mathbf{E}}(\boldsymbol{\xi})+\widehat{\Gamma}^{0}{ }^{(\xi)}\left\{\left(\mathbf{K}^{(2)}-\mathbf{K}^{0}\right) \widehat{\varepsilon}(\boldsymbol{\xi})+\left(\mathbf{K}^{(1)}-\mathbf{K}^{(2)}\right)\right. \\
& \left.\times \sum_{i=1}^{N}\left[\hat{\chi}^{(i)} \hat{\varepsilon}^{\hat{\varepsilon}}\right](\xi)\right\}+\frac{1}{|U|} \hat{\boldsymbol{\Lambda}}^{0}(\boldsymbol{\xi}) \sum_{i=1}^{N} k_{s}^{(i)}\left[\widehat{\mathbf{H}}^{(i)} * \hat{\epsilon}\right](\boldsymbol{\xi}) \\
& -\frac{(d-1)}{|U|} \hat{\boldsymbol{\Lambda}}^{0}(\xi) \sum_{i=1}^{N} k_{s}^{(i)}\left[\widehat{\mathbf{P}}^{(i)} * \hat{\varepsilon}\right](\boldsymbol{\xi}) .
\end{aligned}
$$

The corresponding heat flux field is then determined from the intensity field $\varepsilon(\mathbf{x})$ by

$$
\begin{aligned}
\mathbf{q}(\mathbf{x})= & \mathbf{K}^{(2)} \boldsymbol{\varepsilon}(\mathbf{x})+\left(\mathbf{K}^{(1)}-\mathbf{K}^{(2)}\right) \sum_{i=1}^{N} \chi^{(i)}(\mathbf{x}) \boldsymbol{\varepsilon}(\mathbf{x}) \\
& -\sum_{i=1}^{N} k_{S}^{(i)} \mathbf{x} \nabla_{S} \cdot \varepsilon^{S}(\mathbf{x}) \delta_{T^{(i)}}(\mathbf{x}) .
\end{aligned}
$$

In the transform Fourier space, the heat flux field is given by

$$
\begin{aligned}
\widehat{\mathbf{q}}(\boldsymbol{\xi})= & \mathbf{K}^{(2)} \widehat{\boldsymbol{\varepsilon}}(\boldsymbol{\xi})+\left(\mathbf{K}^{(1)}-\mathbf{K}^{(2)}\right) \sum_{i=1}^{N}\left[\widehat{\chi}^{(i)} * \hat{\boldsymbol{\varepsilon}}\right](\boldsymbol{\xi}) \\
& +\frac{(d-1)}{|U|} \sum_{i=1}^{N} k_{s}^{(i)}\left[\widehat{\mathbf{Q}}^{(i)} * \hat{\varepsilon}\right](\xi)-\frac{1}{|U|} \sum_{i=1}^{N} k_{s}^{(i)}\left[\hat{\mathbb{L}}^{(i)} * \hat{\epsilon}\right](\boldsymbol{\xi}) .
\end{aligned}
$$

where $\hat{\epsilon}_{j l}$ is defined by Eq. (32); $\widehat{\mathbf{Q}}^{(i)}$ and $\widehat{\hat{A}}^{(i)}$ are the second and third-order tensors whose components $\widehat{Q}_{m j}^{(i)}$ and $\widehat{L}_{m l j}^{(l)}$ are specified in Appendices $\mathrm{D}$ and $\mathrm{E}$.

The Eq. (41) for $\widehat{\varepsilon}(\boldsymbol{\xi})$ is solved by using an iterative method in which the numerical algorithm is presented as follows:

\section{- Initialization}

(a) $\hat{\varepsilon}(\xi)=\widehat{\mathbf{E}}(\xi)$

(b) $\hat{\mathbf{q}}^{1}(\xi)=\mathbf{K}^{(2)} \hat{\boldsymbol{\varepsilon}}^{1}(\xi)+\left(\mathbf{K}^{(1)}-\mathbf{K}^{(2)}\right) \sum_{i=1}^{N}\left[\hat{\chi}^{(i)} \hat{*}_{\hat{\varepsilon}}^{1}\right](\xi)$

$$
\begin{aligned}
& +\frac{(d-1)}{|U|} \sum_{i=1}^{N} k_{s}^{(i)}\left[\widehat{\mathbf{Q}}^{(i)} * \hat{\varepsilon}^{-1}\right](\xi) \\
& -\frac{1}{|U|} \sum_{i=1}^{N} k_{s}^{(i)}\left[\hat{\mathbb{L}}^{(i)} * \hat{\boldsymbol{\epsilon}}^{-1}\right](\boldsymbol{\xi}) ;
\end{aligned}
$$

- Iteration $i+1$

(a) Assuming that $\hat{\varepsilon}^{i}(\xi)$ and $\hat{\mathbf{q}}^{i}(\xi)$ are known,

(b) Convergence test,

$$
\begin{aligned}
& \text { (c) } \hat{\varepsilon}^{i+1}(\boldsymbol{\xi})=\widehat{\mathbf{E}}(\boldsymbol{\xi})-\frac{(d-1)}{|U|} \widehat{\boldsymbol{\Lambda}}^{0}(\boldsymbol{\xi}) \sum_{i=1}^{N} k_{s}^{(i)}\left[\widehat{\mathbf{P}}^{(i)} * \hat{\varepsilon}^{i}\right](\boldsymbol{\xi}) \\
& +\widehat{\Gamma}^{0}{ }^{(\xi)}\left\{\left(\mathbf{K}^{(2)}-\mathbf{K}^{0}\right) \hat{\varepsilon}^{i}(\xi)+\left(\mathbf{K}^{(1)}-\mathbf{K}^{(2)}\right)\right. \\
& \left.\times \sum_{i=1}^{N}\left[\hat{\chi}^{(i)} * \hat{\boldsymbol{\varepsilon}}^{i}\right](\xi)\right\}+\frac{1}{|U|} \hat{\boldsymbol{\Lambda}}^{0}(\xi) \sum_{i=1}^{N} k_{\mathrm{s}}^{(i)}\left[\widehat{\mathbf{H}}^{(i)} * \hat{\boldsymbol{\epsilon}}^{i}\right](\xi),
\end{aligned}
$$




$$
\begin{aligned}
& \text { (d) } \hat{\mathbf{q}}^{i+1}(\boldsymbol{\xi})=\mathbf{K}^{(2)} \hat{\mathcal{\varepsilon}}^{i+1}(\boldsymbol{\xi})+\left(\mathbf{K}^{(1)}-\mathbf{K}^{(2)}\right) \sum_{i=1}^{N}\left[\hat{\chi}^{(i)} *_{\bar{\varepsilon}}^{i+1}\right](\boldsymbol{\xi}) \\
& +\frac{(d-1)}{|U|} \sum_{i=1}^{N} k_{s}^{(i)}\left[\widehat{\mathbf{Q}}^{(i)} *_{\varepsilon}^{i+1}\right](\xi) \\
& -\frac{1}{|U|} \sum_{i=1}^{N} k_{s}^{(i)}\left[\hat{\mathbb{L}}^{(i)} *_{\epsilon}^{\hat{\epsilon} \epsilon+1}\right](\xi) .
\end{aligned}
$$

Additionally, the convergence of the iterative computation is reached or equivalently the iterative procedure is stopped when

$$
\frac{\left\|\hat{\mathbf{q}}^{i+1}(\boldsymbol{\xi})-\hat{\mathbf{q}}^{i}(\boldsymbol{\xi})\right\|}{\left\|\hat{\mathbf{q}}^{i+1}(\boldsymbol{\xi})\right\|}<\gamma,
$$

where $\gamma$ is a prescribed value which set at 0.001 in our calculations.

The macroscopic heat flux defined as Eq. (16) or (17) can be determined from the Fourier transform of the local heat flux field by setting the wave vector equal to zero in Eq. (43), i.e.,

$$
\begin{aligned}
\mathbf{Q}= & \mathbf{K}^{(2)} \hat{\varepsilon}(\mathbf{0})+\left(\mathbf{K}^{(1)}-\mathbf{K}^{(2)}\right) \sum_{i=1}^{N}\left[\hat{\chi}^{(i)} * \hat{\varepsilon}\right](\mathbf{0}) \\
& +\frac{(d-1)}{|U|} \sum_{i=1}^{N} k_{s}^{(i)}\left[\widehat{\mathbf{Q}}^{(i)} * \hat{\varepsilon}\right](\mathbf{0})-\frac{1}{|U|} \sum_{i=1}^{N} k_{s}^{(i)}\left[\widehat{\mathbb{L}}^{(i)} * \hat{\epsilon}\right](0) .
\end{aligned}
$$

Thus, by setting successively the macroscopic intensity component $E_{j}=1$ with $j=1,2, \ldots, d$ and by using the effective behavior described by Eq. (14), the effective thermal conductivity tensor components are then given by $K_{i j}^{\text {eff }}=Q_{i}$.

\section{Numerical examples}

\subsection{Fiber-matrix composites}

To numerically illustrate the features of the results obtained above, we consider the first example of a fiber composite submitted to a heat flow normal to the direction of the fibers. The fibers are assumed cylindrical with circular sections having the same radius, i.e. $R_{i}=R$, and introduced periodically into the host matrix phase. For this 2D problem, three typical microstructures with squared, hexagonal and random distributions of the inhomogeneities are studied in detail. In addition, the inhomogeneity phase is assumed to be less conducting than the matrix one. The thermal conductivities of the matrix and inhomogeneities are chosen as:

$k_{1}=0.1 \mathrm{Wm}^{-1} \mathrm{~K}^{-1}, \quad k_{2}=1 \mathrm{Wm}^{-1} \mathrm{~K}^{-1}$.

Next, to define the thermal parameters of the interface $\Gamma^{(i)}$ between the ith-inhomogeneity and matrix phases, we start with the interphase layer in which the intermediate medium between the $i$ th-inhomogeneity and matrix phase are assumed to be very thin and very highly conducting. The corresponding thickness $h^{(i)}$ and conductivity $k_{c}^{(i)}$ are chosen as $k_{c}^{(i)}=50 \mathrm{~W} \mathrm{~m}^{-1} \mathrm{~K}^{-1}$ and $\mathrm{h}^{(i)}=20 \mathrm{~nm}$

Then, from the asymptotic approach (see e.g. $[1,4,14,28,30]$ ), it can be shown that this thin and highly conducting layer can be appropriately modeled as an imperfect coherent interface with the surface thermal conductivity given by

$k_{s}^{(i)}=h^{(i)} k_{c}^{(i)}=10^{-6} \mathrm{Wm}^{-1} \mathrm{~K}^{-1}$.

Additionally, the thermal conductivity tensor of the reference medium is given by $\mathbf{K}^{0}=k^{0} \mathbf{I}^{(d)}$ with $k^{0}=k_{c}^{(i)}=50 \mathrm{~W} \mathrm{~m}{ }^{-1} \mathrm{~K}^{-1}$

Next, in order to analyze the dependence of the effective thermal conductivity of the composite on the size and distribution of the inhomogeneities, the radius $R$ of the inhomogeneities inside the host matrix media is varied from 1 to $50 \mu \mathrm{m}$ while the inhomogeneities fraction $c_{1}$ is kept constant with $c_{1}=0.3$ for three cases with squared, hexagonal and random distributions of the inhomogeneities. The case of random inclusions is studied with 5 different simulations, each containing 100 inclusions within each period and the results presented thereafter correspond to the mean value, to the minimum values and maximal values obtained from the 5 simulations.

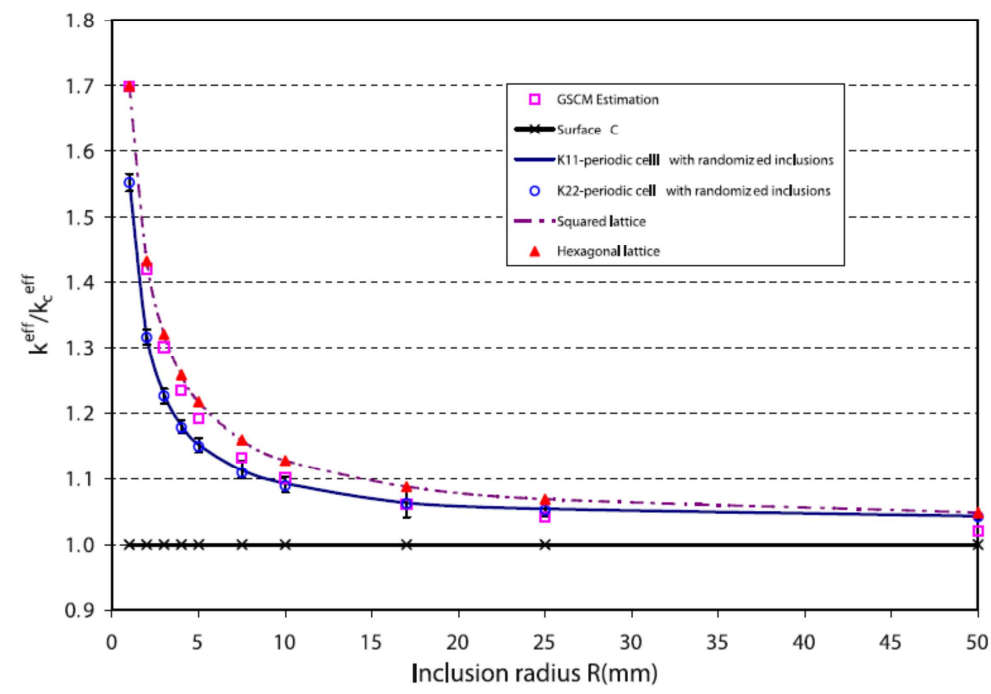

Fig. 3. Dependencies of the ratio $k^{\mathrm{eff}} / k c^{e f f}$ on the circular inclusion radius $\mathrm{R}(\mu \mathrm{m})$ and on the distribution of inclusions with inclusion fraction $c_{1}=0.3$ and $N_{\mathrm{k}}=64^{*} 64$. 


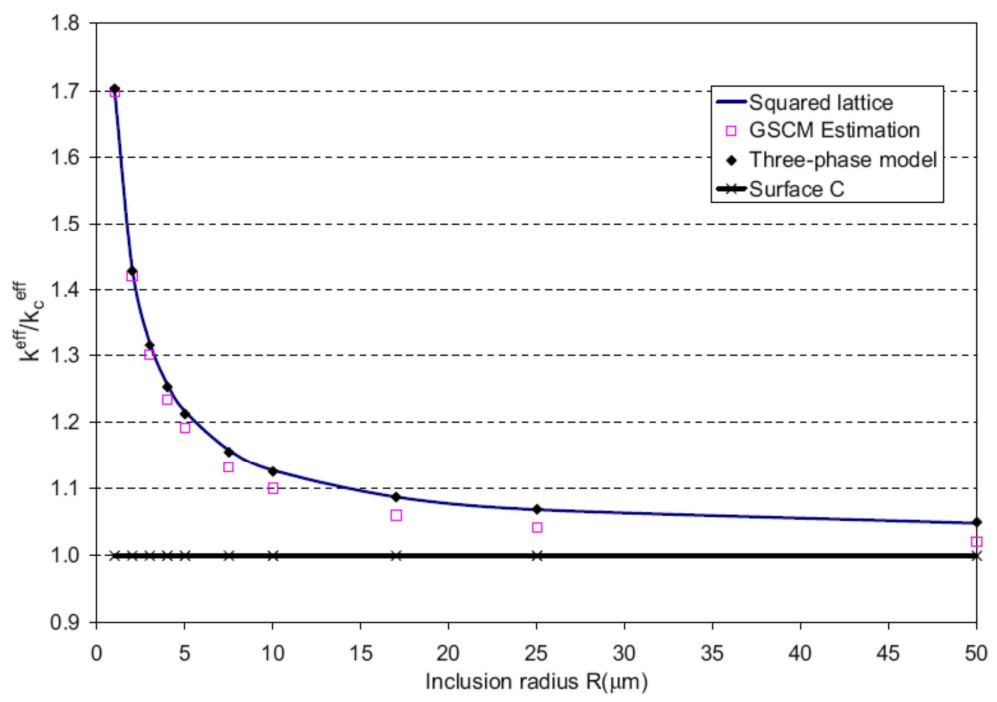

Fig. 4. The ratio $k^{\text {eff }} / k_{c}^{\text {eff }}$ versus the circular inclusion radius $R(\mu \mathrm{m})$ with inclusion fraction $c_{1}=0.3$ and $N_{k}=64 \times 64$.

The values of ratio $k_{i j}^{e f f} / k_{c i j}^{\text {eff }}$ are plotted in Fig. 3 in terms of the inhomogeneity radius $R$ with $c_{1}=0.3$, respectively, where $k_{i j}^{\text {eff }}$ is the component of the effective thermal conductivity tensor according to the coherent interface model and $k_{c i j}^{e f f}$ denotes the component of the effective thermal conductivity tensor without accounting for the surface effects, i.e. for a continuous normal flux (surface $\mathrm{C}$ ). The obtained numerical values for $k_{i j}^{\text {eff }} / k_{c i j}^{\text {eff }}$ are then compared with the ones derived by applying the generalized self-consistent model. To get more details for the latter model, the reader can refer to Appendix B. For each result, only the effect of the interface is studied. For this reason, each result is divided by the value of $k_{c i j}^{e f f}$ related to the same kind of modeling.

It can be seen from Fig. 3 that: (i) the effective conductivity behavior of both cases with and without accounting for the surface effects of periodic composite with squarely and hexagonally distributed inclusions are isotropic, i.e. $k_{11}^{\text {eff }}=k_{22}^{\text {eff }}=k^{\text {eff }}$ and $k_{11 c}^{\text {eff }}=k_{22 c}^{\text {eff }}=k_{c}^{\text {eff }}$. A very small difference between the results related to both directions can be observed for the periodic composite with randomly distributed inclusions after 5 realizations, but the behavior is also practically isotropic in this case.

(ii) $k^{\text {eff }}$ depends on the inhomogeneity radius $R$, which corresponds to the size effect observed in the literature, while $k_{c}^{\mathrm{eff}}$ is independent of $R$, meaning that the effective behavior without surface energy does not display a size effect. The effective conductivity of the composite with $\mathrm{HC}$ interfaces can reach

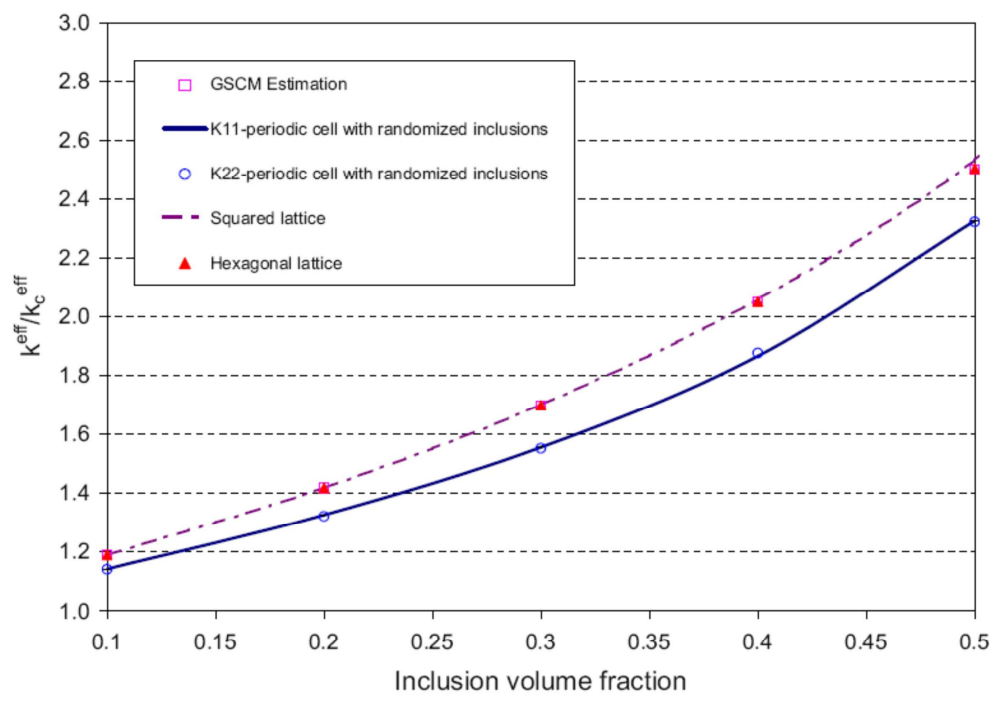

Fig. 5. The ratio $k^{\text {eff }} / k_{c}^{\text {eff }}$ versus the inclusion fraction $c_{1}$ with circular inclusion radius $R=1 \mu \mathrm{m}$ and $N_{k}=64 \times 64$. 


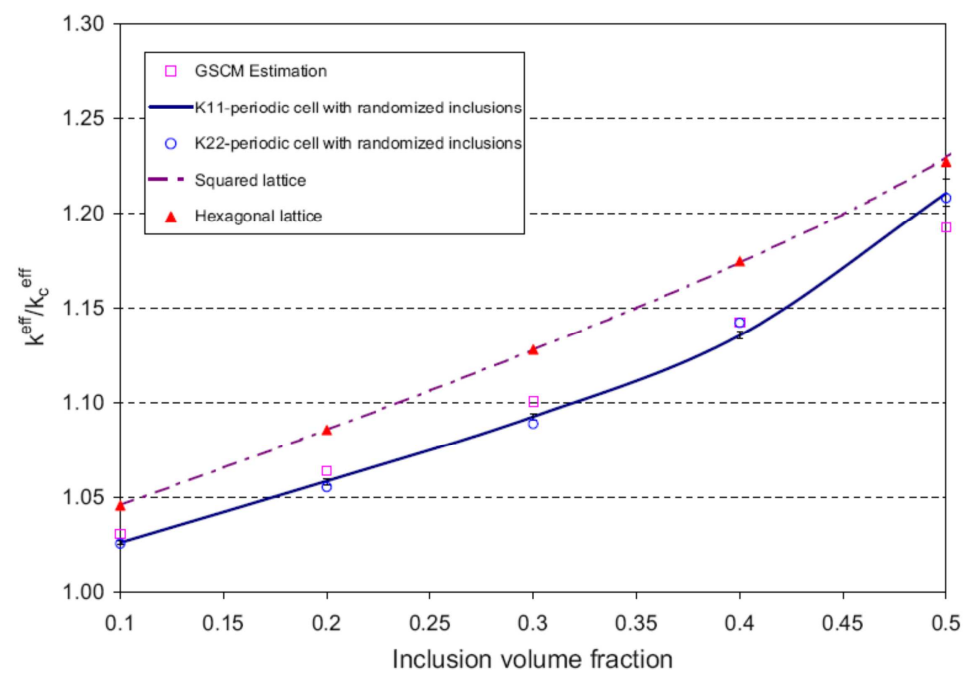

Fig. 6. The ratio $k^{\text {eff }} / k_{c}^{\text {eff }}$ versus the inclusion fraction $c_{1}$ with circular inclusion radius $R=10 \mu \mathrm{m}$ and $N_{k}=64 \times 64$.

nearly 2 times the value without interface effect, for a radius being around $1 \mu \mathrm{m}$.

(iii) the difference between $k^{\text {eff }}$ and $k_{c}^{\text {eff }}$ decreases when $R$ increases, which means that for a same concentration, the surface energy increases when the heterogeneity size decreases.

(iv) the surface effect becomes small (a few percent) when $R$ is larger than $50 \mu \mathrm{m}$;

(v) the values $k^{\text {eff }} / k_{c}^{e f f}$ obtained by employing the coherent interface model are consistent with the estimation given by using the generalized self-consistent model.
To check the consistence of the coherent interface model described above, we compare in Fig. 4 the values for $k^{\text {eff }} / k_{c}^{\text {eff }}$ related to the squared lattice periodic composite with the ones derived by using a periodic three-phase model with a squared lattice, it means a model with perfect interfaces, but containing an interphase between inclusions and matrix which is equivalent with the imperfect interface. The numerical values show an excellent agreement between both configurations.

Finally, the ratios $k_{i j}^{\text {eff }} / k_{\text {cfij }}^{\text {eff }}$ in terms of the fraction of inclusions $c_{1}$ are depicted in Figs. 5 and 6 with two inclusion radii $R=1 \mu \mathrm{m}$ and $R=10 \mu \mathrm{m}$. As expected, the surface effect increases for higher

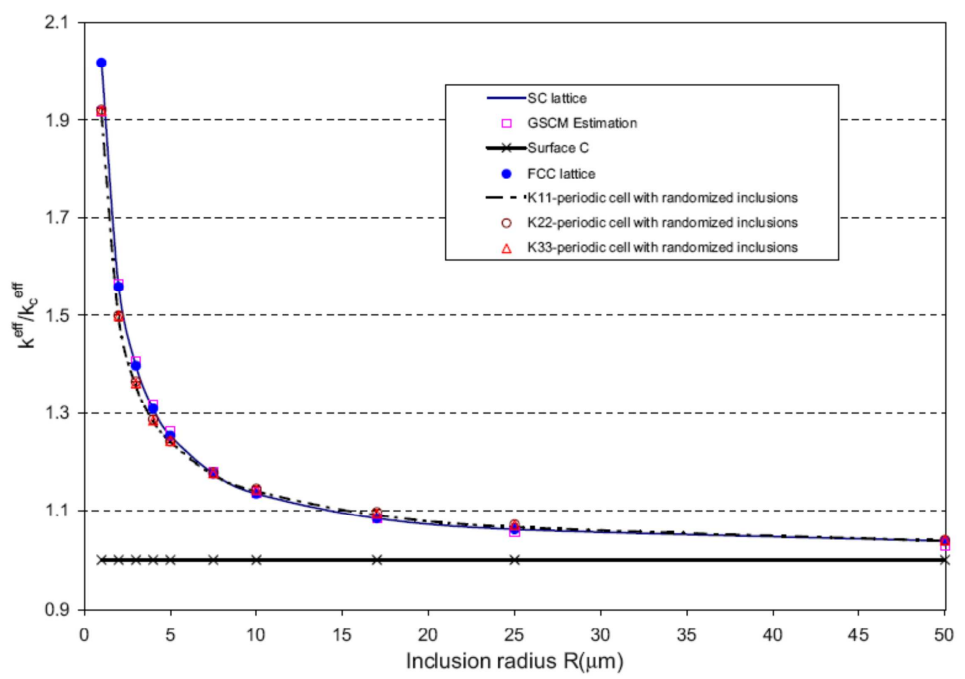

Fig. 7. Dependencies of the ratio $k^{\text {eff }} / k_{c}^{\text {eff }}$ on the spherical inclusion radius $R(\mu \mathrm{m})$ and on the distribution of inclusions with inclusion fraction $c_{1}=0.3$ and $N_{k}=64 \times 64 \times 64$. 


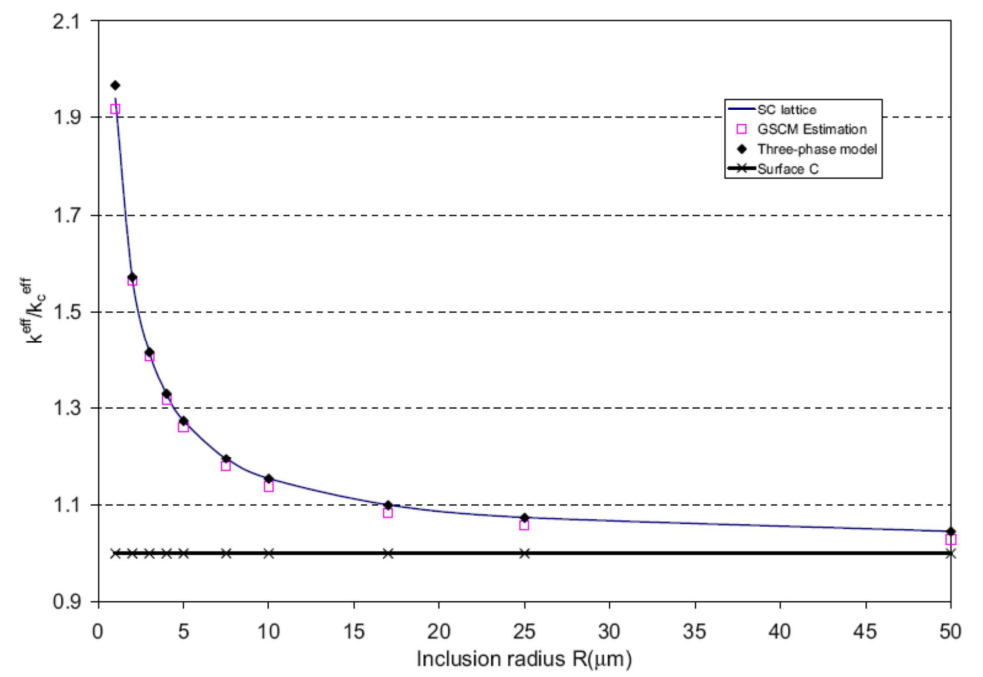

Fig. 8. The ratio $k^{\text {eff }} / k_{c}^{\text {eff }}$ versus the spherical inclusion radius $R(\mu \mathrm{m})$ with inclusion volume fraction $c_{1}=0.3$ and $N_{k}=64 \times 64 \times 64$.

values of $c_{1}$ and can reach more than two times the value without interface effect for higher concentrations of inclusions. Given a value of the inclusion fraction, the surface effect becomes more important when the inclusion radius diminishes. From these figures, it can also be seen that the size effect is similar for the case of hexagonal and squared lattices. The cases of random distributions produce a size effect which is similar to the one of the GSCM estimation.

\subsection{Particle-matrix composites}

The second example consists of a 3D host matrix in which spherical inclusions are embedded. As in the case 2D, all spherical inclusions are assumed to have the same radius, $R_{i}=R$, and the thermal parameters of matrix and inclusions phases as well as the interfaces between the matrix and inclusions phases are the same as those of the example of fiber-matrix composite. Similarly to the case $2 \mathrm{D}$, for periodic composite with randomly distributed inclusions, the number of realizations is equal to 5 . However, the number of spherical inclusions for a unit cell is now equal to 10 , which produces a discrepancy similar to the one obtained in the case of the fiber-matrix composite.

The effect of the inclusion size on the effective thermal conductivity is shown in Figs. 7 and 8 for the periodic composite with simple cubic (SC), face centered cubic (FCC) and randomly distributed inclusions. The radius $R$ of the spherical inhomogeneities increases

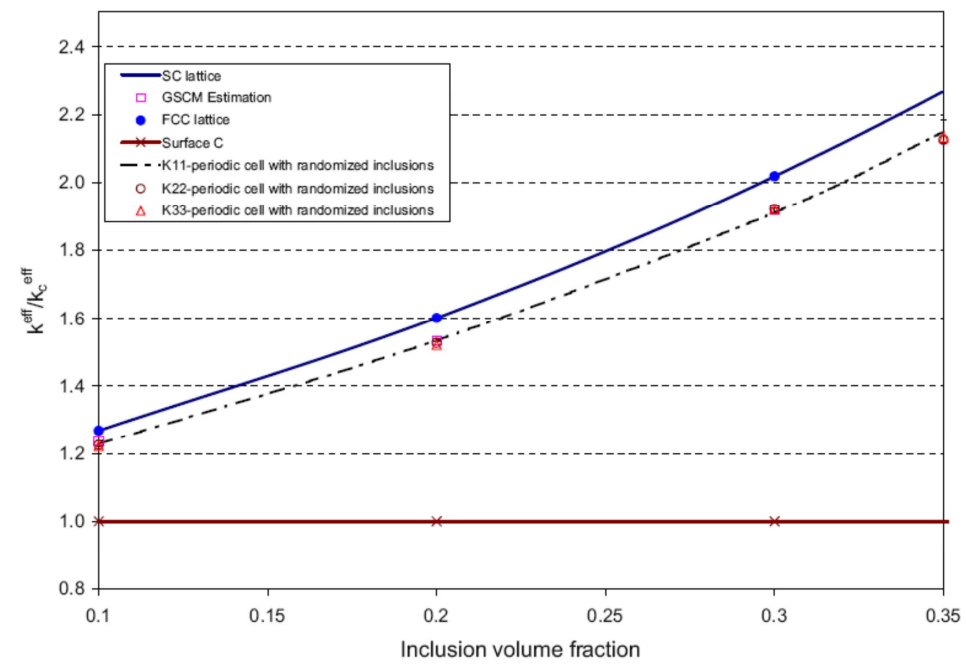

Fig. 9. The ratio $k^{\text {eff }} / k_{c}^{\text {eff }}$ versus the inclusion fraction $c_{1}$ with spherical inclusion radius $R=1 \mu \mathrm{m}$ and $N_{k}=64 \times 64 \times 64$ 


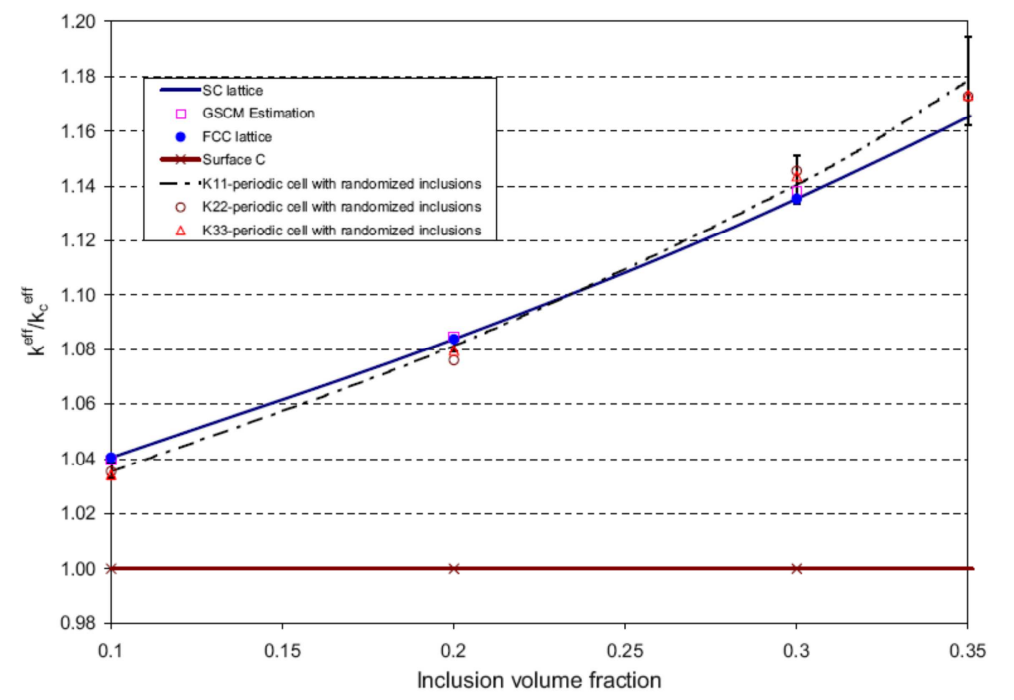

Fig. 10. The ratio $k^{\text {eff }} / k_{c}^{\text {eff }}$ versus the inclusion fraction $c_{1}$ with spherical inclusion radius $R=10 \mu \mathrm{m}$ and $N_{k}=64 \times 64 \times 64$.

from 1 to $50 \mu \mathrm{m}$ while the inhomogeneity volume fraction $c_{1}$ is given constant and equal to 0.3 . The results are similar to those obtained in the case of the fiber-matrix composite. However, in the case of the particle-matrix composite, the size effect is clearly more significant than in the previous case. Inversely, the effect of the distribution of particles on the effective properties is very small compared to analogous cases in the case of the fiber-matrix composite.

These numerical values for the size-dependent effective thermal conductivity are then compared to the ones obtained by applying the three-phase model and generalized self-consistent model. Taking the inclusion radius to be equal to $R=1 \mu \mathrm{m}$ and $R=10 \mu \mathrm{m}$, the ratio $k_{i j}^{e f f} / k_{c i j}^{e f f}$ is plotted versus the inclusion fraction volume $c_{1}$ in Figs. 9 and 10. As for the case of inclusion-matrix composites, the case of small inclusions shows that the size effect is similar for both periodic cases while the cases of random distributions produce results similar to the one obtained from the GSCM model.

\subsection{Convergence study}

All results presented can be affected by the choice of two numerical parameters: the value of the reference medium used for the computation of the Green's function and the quantity of wave numbers used within the Fourier transform. Without surface effect, the mean value between the properties of the matrix and of the

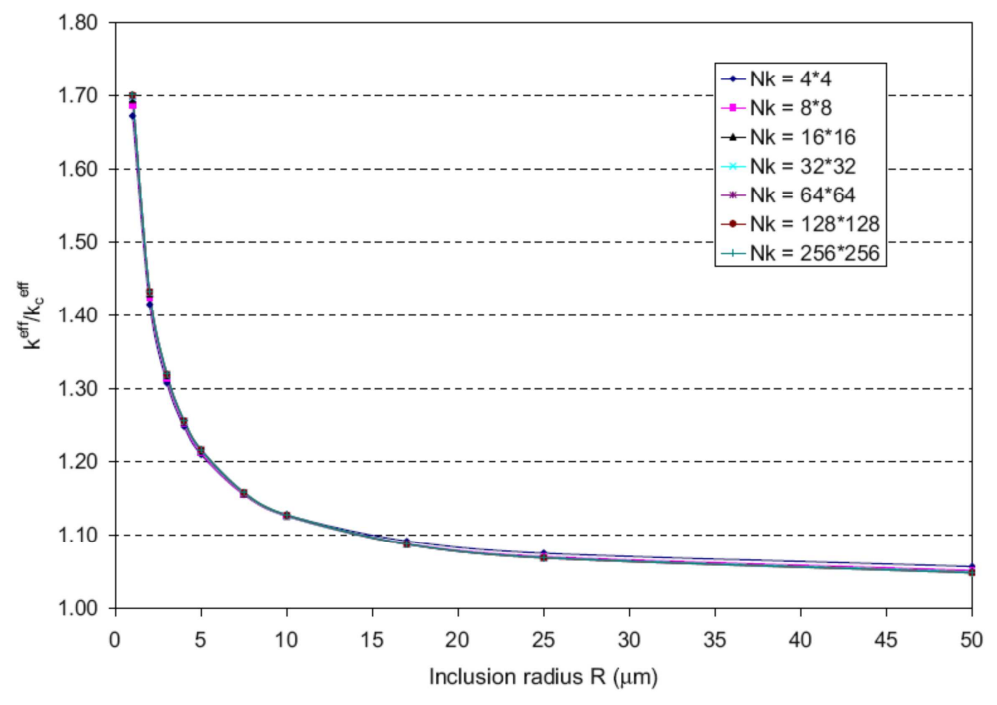

Fig. 11. Study of convergence in wave numbers with circular inclusion fraction $c_{1}=0.3$. 


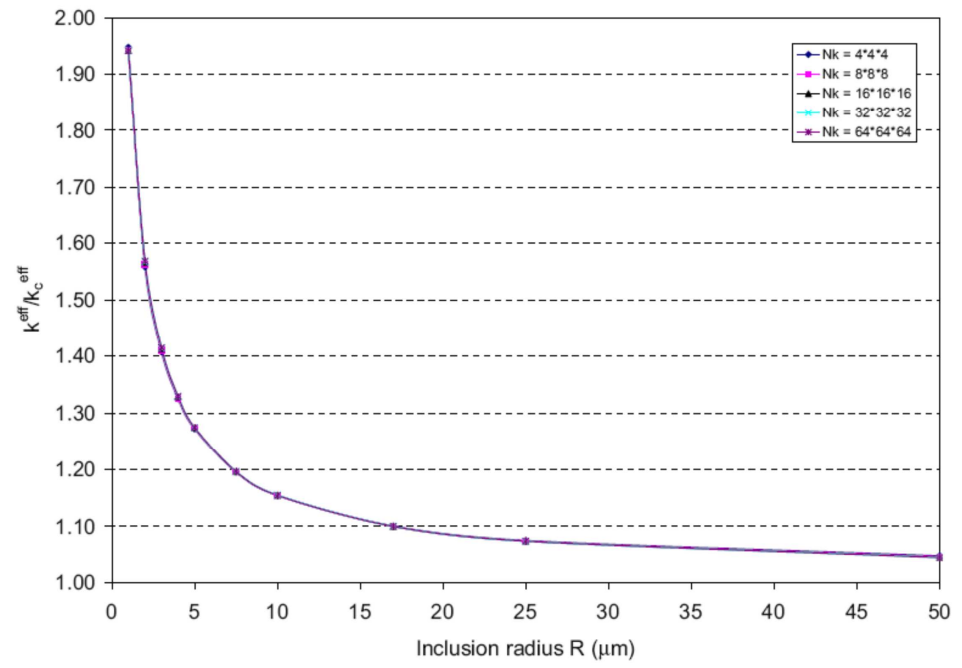

Fig. 12. Study of convergence in wave numbers with spherical inclusion fraction $c_{1}=0.3$.

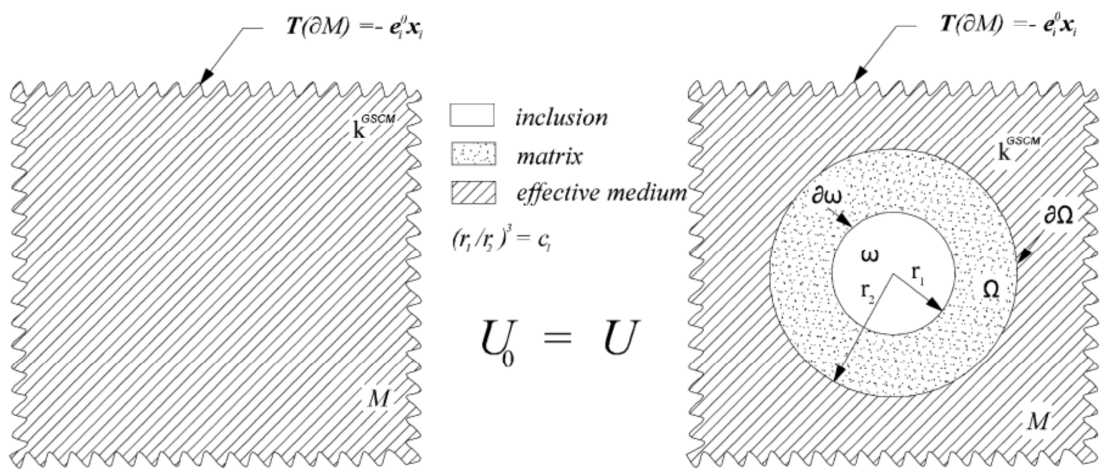

Fig. 13. Generalized self-consistent model applied to thermal conduction problem.

inclusions is convenient. Taking into account the HC surface effect needs to increase significantly the conductivity of the reference medium, as shows the value previously given. The effect of the quantity of wave number $N_{k}$ on the effective thermal conductivity for squared lattice periodic composite accounting to the coherent interface model is illustrated in Fig. 11 for the case 2D and Fig. 12 for the case 3D. It can be seen from Figs. 11 and 12 that the obtained numerical values for $k^{\text {eff }} / k_{c}^{\text {eff }}$ decrease continuously when the wave number $N_{k}$ increases. Additionally, Figs. 11 and 12 show also that a number of wave vectors of 64 in each direction is convenient to reach the convergence for both $2 \mathrm{D}$ and $3 \mathrm{D}$ cases.

\section{Conclusions}

The effective conductivity of periodic composites with highly conducting imperfect interfaces has been studied by using a solution in Fourier space and the known Fourier transform of the Green's tensor for conductivity. The plane problem of periodic fiber-matrix composites with squared lattice, hexagonal lattice and periods containing random distributions so as the 3D problem of particle-matrix composites with cubic SC or FCC lattices and random distributions were studied. The results show that the effect of imperfect $\mathrm{HC}$ interfaces can increase significantly the effective conductivity of the medium when the heterogeneity size becomes small, displaying a significant size effect. The size effect is found to be comparable for periodic cases, the case of random distributions leading to size effects similar to those of the Generalized Self Consistent Model. The dual case of Kapitza interfaces related to highly resistive interfaces is currently under study.

\section{Appendices}

A. Derivation of equation (22)

First, by using the definition (30) of the surface divergence, we have 


$$
\nabla_{S} \cdot(\mathbf{P v})=\operatorname{Tr}[\nabla(\mathbf{P v}) \mathbf{P}]
$$

for any differentiable vector $\mathbf{v}$. Using the suffix notation, the last term of (1) can be rewritten in the equivalent form

$$
\begin{aligned}
\operatorname{Tr}[\nabla(\mathbf{P v}) \mathbf{P}] & =\left(P_{i k} v_{k}\right)_{{ }_{m}} P_{m i}=v_{k} P_{i k, m} P_{m i}+v_{k, m} P_{m i} P_{i k} \\
& =v_{k}\left(\delta_{i k}-n_{i} n_{k}\right)_{, m} P_{m i}+v_{k, m} P_{m k}^{2} \\
& =-v_{k}\left(n_{i, m} n_{k}+n_{i} n_{k, m}\right) P_{m i}+v_{k, m} P_{m k}^{2} \\
& =-(\mathbf{v} \cdot \mathbf{n}) \operatorname{Tr}[\mathbf{P} \nabla \mathbf{n}]-(\nabla \mathbf{n} \mathbf{P n}) \cdot \mathbf{v}+\operatorname{Tr}\left[\nabla \mathbf{v} \mathbf{P}^{2}\right] .
\end{aligned}
$$

Next, due to the fact that the orthogonal complementary projection operator $\mathbf{P}$ defined by Eq. (5) have following properties

$$
\mathbf{P}^{2}=\mathbf{P}, \quad \mathbf{P} \cdot \mathbf{n}=0,
$$

then Eq. (A2) becomes

$\operatorname{Tr}[\nabla(\mathbf{P v}) \mathbf{P}]=-(\mathbf{v} \cdot \mathbf{n}) \operatorname{Tr}[\mathbf{P} \nabla \mathbf{n}]+\operatorname{Tr}[\nabla \mathbf{v} \cdot \mathbf{P}]$.

Moreover, when the $i$ th-inclusion is 3D spherical or 2D circular of radius $R_{i}$, it is easy to show that

$$
\nabla \mathbf{n}=\frac{1}{R_{i}} \mathbf{P}
$$

Substituting Eq. (A5) into Eq. (A4) and accounting for the fact that $\operatorname{Tr}\left(\mathbf{P}^{2}\right)=\operatorname{Tr}(\mathbf{P})=d-1$, Eq. (A4) is reduced to

$$
\operatorname{Tr}[\nabla(\mathbf{P v}) \mathbf{P}]=-\frac{(d-1)}{R_{i}} \mathbf{v} \cdot \mathbf{n}+\operatorname{Tr}[\nabla \mathbf{v} \mathbf{P}] .
$$

Finally, by combining Eq.(A6) with Eq.(1) together with Eq. (30), we derive the equality (22), i.e.,

$\nabla_{S} \cdot(\mathbf{P v})=-\frac{(d-1)}{R_{i}} \mathbf{v} \cdot \mathbf{n}+\nabla_{S} \cdot \mathbf{v}$

B. Generalized self-consistent model

The model presented in this appendix can be considered as an extension to the thermal conduction problem with interface effects of the classical GSCM for elasticity which was initiated by Van der Poel [34], improved and completed by Smith $[32,33]$ and Christensen and Lo [10].

Relative to this model, we first consider an infinite threedimensional medium $M$ consisting of the effective homogeneous and isotropic medium whose thermal behavior is characterized by Eq. (14) in which the effective thermal conductivity tensor is denoted by $\mathbf{K}^{\text {eff }}=k^{G S C M} \mathbf{I}^{(3)}$. As before, let $M$ now be subjected to the uniform boundary conditions $T(\mathbf{x})=-\mathbf{E} \cdot \mathbf{x}$ with any $\mathbf{x} \in \partial M$ and the constant intensity vector $\mathbf{E}$ chosen such as $\mathbf{E}^{0}=\left(0,0, e^{0}\right)^{T}$ with $e^{0}$ being a constant intensity field (Fig. 13). In the system of spherical coordinates $(r, \theta, \phi)$ corresponding to the spherical orthogonal basis $\left(\mathbf{f}_{r}, \mathbf{f}_{\theta}\right.$, $\mathbf{f}_{\phi}$ ), this uniform boundary condition takes the equivalent form

$T^{0}(\mathbf{x})=-e^{0} r \cos \theta, \quad \mathbf{x} \in \partial M$.

Because the material constituting medium $M$ is homogeneous and isotropic, this boundary condition produces the following temperature, intensity and heat flux fields in $M$

$T^{0}(\mathbf{x})=e^{0} r \cos \theta, \quad \mathbf{e}^{0}(\mathbf{x})=e^{0}\left(\cos \theta \mathbf{f}_{r}-\sin \theta \mathbf{f}_{\theta}\right)$, $\mathbf{q}^{0}(\mathbf{x})=k^{G S C M} e^{0}\left(\cos \theta \mathbf{f}_{r}-\sin \theta \mathbf{f}_{\theta}\right)$.

Let us introduce the following virtual work $U_{0}\left(\mathbf{e}^{0}\right)$ of $M$ (see e.g. [13])

$U_{0}\left(\mathbf{e}^{0}\right)=\int_{M} \mathbf{q}^{0}(\mathbf{x}) \cdot \mathbf{e}^{0}(\mathbf{x}) \mathrm{d} \mathbf{x}=\operatorname{vol}(M) k^{G S C M}\left(e^{0}\right)^{2}$.
Next, we cut a sphere out of the foregoing infinite effective medium and substitute back a composite sphere $\Omega$ while imposing the same boundary condition on $\partial M$ as before. The interface between the composite sphere and the outside medium is assumed to be perfect. The core of this composite sphere is made of the inclusion phase and surrounded by a concentric shell consisting of the matrix phase. The core and outer coating consist of two isotropic materials whose thermal conductivities are $k_{1}$ and $k_{2}$. The radii of the core and coating, symbolized by $r_{1}$ and $r_{2}$ are chosen so as to be compatible with the prescribed phase volume fraction

$c_{1}=1-c_{2}=\frac{r_{1}^{3}}{r_{2}^{3}}$.

The 3D spherical interface $\Gamma$ between the matrix and inclusion is modeled by the HC surface model as described in Section 2.

Under the boundary condition Eq. (B1), the expressions of the temperature field, non-zero intensity and heat flux field components are given by (see, e.g. [15])

$T^{(i)}=-\left(a_{i} r+\frac{b_{i}}{r^{2}}\right) \cos \theta$,

$e_{r}^{(i)}=\left(a_{i}-\frac{2 b_{i}}{r^{3}}\right) \cos \theta, \quad e_{\theta}^{(i)}=-\left(a_{i}+\frac{b_{i}}{r^{3}}\right) \sin \theta$,

$q_{r}^{(i)}=k_{i}\left(a_{i}-\frac{2 b_{i}}{r^{3}}\right) \cos \theta, \quad q_{\theta}^{(i)}=-k_{i}\left(a_{i}+\frac{b_{i}}{r^{3}}\right) \sin \theta$.

with $i=1$ referring to the core, $i=2$ to the outer coating and $i=e$ to the external effective medium. In these expressions, $a_{i}$ and $b_{i}$ are constants to be determined from the boundary and interface conditions together with a condition avoiding the displacement singularity in the core of the composite sphere. More precisely, the requirement of the temperature in the core at $r=0$ to be finite implies that $b_{1}=0$. The value of $a_{e}$, determined by using the boundary condition (B1) with $r \rightarrow \infty$, is given by $a_{e}=e^{0}$.

At the interface between the core and the coating, the temperature and tangential part of the intensity fields are continuous, so that

$a_{1}=a_{2}+\frac{b_{2}}{r_{1}^{3}}$

$e_{\theta}^{S}=e_{\theta}^{(1)}=e_{\theta}^{(2)}=-\left(a_{2}+\frac{b_{2}}{r_{1}^{3}}\right) \sin \theta$,

$e_{\varphi}^{S}=e_{\varphi}^{(1)}=e_{\varphi}^{(2)}=0$.

The surface condition (8) at the interface between the core and the coating can be specified by accounting for Eqs. (7), (9), (B9) and (B7) and reduced to the following one:

$k_{2}\left(a_{2}-\frac{2 b_{2}}{r_{1}^{3}}\right)-k_{1} a_{1}=2 \widehat{k}_{s}\left(a_{2}+\frac{b_{2}}{r_{1}^{3}}\right)$,

with $\widehat{k}_{s}=k_{s} / r_{1}$.

At the same time, the interface at $r=r_{2}$ between the coating matrix and outside effective medium is perfectly bonded. Thus, the continuity conditions of the temperature field $T$ and normal component heat flux field $q_{\mathrm{r}}$ across the interface at $r=r_{2}$ are expressed as

$e^{0}+\frac{b_{e}}{r_{2}^{3}}=a_{2}+\frac{b_{2}}{r_{2}^{3}}$ 
$k^{\operatorname{GSCM}}\left(e^{0}-\frac{2 b_{e}}{r_{2}^{3}}\right)=k_{2}\left(a_{2}-\frac{2 b_{2}}{r_{2}^{3}}\right)$.

As in the GSCM of Christensen and Lo [10] in the context of elasticity, the effective thermal conductivity is required to be such that the virtual work $U\left(\mathbf{e}^{0}\right)$ after introducing the sphere composite is equal to the initial one $U_{0}\left(\mathbf{e}^{0}\right)$ which is given by Eq. (B3). On the other hand, we can show that the actual virtual work $U\left(\mathbf{e}^{0}\right)$ can be expressed in the terms of $U_{0}\left(\mathbf{e}^{0}\right)$ as follows

$U=U_{0}+\int_{\Gamma}\left(\mathbf{q}^{0} T^{(e)}-\mathbf{q}^{(e)} T^{0}\right) \cdot \mathbf{n d} \mathbf{x}$.

Thus, the self-consistency condition $U_{0}=U$ is reduced to

$\int_{\Gamma}\left(\mathbf{q}^{0} T^{(e)}-\mathbf{q}^{(e)} T^{0}\right) \cdot \mathbf{n d} \mathbf{x}=0$.

Substituting Eqs. (B1), (B2), (B5) and (B7) with $i=e$ into Eq. (B14), we obtain the simple equation

$b_{e}=0$.

Finally, substituting $b_{e}=0$ into Eqs. (B8), (B10)-(B12), we obtain a system of four homogeneous linear equations for the four unknowns $a_{1}, a_{2}, b_{2}$ and $e^{0}$. A non-trivial solution to this system exists if and only if the determinant of the relevant $4 \times 4$ matrix is equal to zero. This necessary and sufficient condition yields the expression for the effective thermal conductivity as follows:

$k^{\mathrm{GSCM}}=k_{2}+\frac{3 k_{2} c_{1}\left(k_{1}-k_{2}+2 \widehat{k}_{s}\right)}{3 k_{2}+c_{2}\left(k_{1}-k_{2}+2 \widehat{k}_{s}\right)}$.

Firstly, we remark that the effective thermal conductivity obtained by Eq. (B16) depends not only on the phase thermal properties and volume fractions but also on the interface thermal conductivity and on the size of the inhomogeneities through $k_{\mathrm{s}}$.

In the $2 \mathrm{D}$ case, the corresponding effective thermal conductivity is given by

$k^{G S C M}=k_{2}+\frac{2 k_{2} c_{1}\left(k_{1}-k_{2}+\widehat{k}_{s}\right)}{2 k_{2}+c_{2}\left(k_{1}-k_{2}+\widehat{k}_{s}\right)}$.

The details of the derivation are omitted here.
Finally, it is convenient to express (B16) and (B17) for $k^{G S C M}$ by the following compact one:

$k^{\mathrm{GSCM}}=k_{2}+\frac{d k_{2} c_{1}\left[k_{1}-k_{2}+(d-1) \hat{k}_{s}\right]}{d k_{2}+c_{2}\left[k_{1}-k_{2}+(d-1) \widehat{k}_{s}\right]}$,

where $d(=3$ or 2$)$ is the dimension of the problem.

C. The expressions for the components $\widehat{H}_{m j}^{(i)}\left(\xi-\xi^{\prime}\right)$ of matrix $\widehat{\mathbf{H}}^{(i)}\left(\boldsymbol{\xi}-\xi^{\prime}\right)$ and components $\widehat{P}_{m}^{(i)}\left(\boldsymbol{\xi}-\xi^{\prime}\right)$ of vector $\mathbf{P}^{(i)}\left(\boldsymbol{\xi}-\xi^{\prime}\right)$ in Eq. (31)

(i) when the composite with circular ith-inclusion of radius $R_{i}$ is under consideration

$$
\begin{aligned}
& \widehat{H}_{11}^{(i)}= \begin{cases}2 \iota \pi R_{i}\left[\frac{J_{1}(z)}{z}-s^{2} J_{2}(z)\right] e^{-\imath\left(\xi-\xi^{\prime}\right) \cdot X^{(i)}} & \text { for } z \neq 0 ; \\
\iota \pi R_{i} e^{-\iota\left(\xi-\xi^{\prime}\right) \cdot \mathbf{X}^{(i)}} & \text { for } z=0 ;\end{cases} \\
& \widehat{H}_{22}^{(i)}= \begin{cases}2 \iota \pi R_{i}\left(\frac{J_{1}(z)}{z}-c^{2} J_{2}(z)\right) e^{-\iota\left(\xi-\xi^{\prime}\right) \cdot \mathbf{X}^{(i)}} & \text { for } z \neq 0 ; \\
\iota \pi R_{i} e^{-\iota\left(\xi-\xi^{\prime}\right) \cdot \mathbf{X}^{(i)}} & \text { for } z=0 ;\end{cases}
\end{aligned}
$$

$\widehat{H}_{12}^{(i)}=\widehat{H}_{21}^{(i)}= \begin{cases}2 \iota \pi R_{i} c S J_{2}(z) e^{-\imath\left(\xi-\xi^{\prime}\right) \cdot \mathbf{X}^{(i)}} & \text { for } z \neq 0 \\ 0 & \text { for } z=0\end{cases}$

$$
\begin{aligned}
& \widehat{P}_{1}^{(i)}= \begin{cases}-2 \iota \pi c J_{1}(z) e^{-\imath\left(\xi-\xi^{\prime}\right) \cdot \mathbf{X}^{(i)}} & \text { for } z \neq 0 \\
0 & \text { for } z=0 ;\end{cases} \\
& \widehat{P}_{2}^{(i)}= \begin{cases}-2 \iota \pi S J_{1}(z) e^{-\imath\left(\xi-\xi^{\prime}\right) \cdot \mathbf{X}^{(i)}} & \text { for } z \neq 0 \\
0 & \text { for } z=0\end{cases}
\end{aligned}
$$

where $c=\cos \theta_{0}$ and $s=\sin \theta_{0}$ with $\theta_{0}$ standing for the angle between the discrete vector $\xi-\xi^{\prime}$ and the unit vector $\mathbf{f}_{1}$

(ii) when the composite with spherical $i$ th-inclusion of radius $R_{i}$ is concerned

$$
\begin{aligned}
& \hat{H}_{11}^{(i)}= \begin{cases}-\frac{4 \imath \pi R_{i}^{2}\left[-z c s_{2}^{2}-c_{1}^{2} c_{2}^{2}(z c-s)-z^{2} s+s_{2}^{2} s+2 z c c_{2}^{2} s_{1}^{2}+c_{2}^{2} s_{1}^{2} s\left(z^{2}-2\right)\right]}{z^{3}} e^{-l\left(\xi-\xi^{\prime}\right) \cdot \mathbf{X}^{(i)}} & \text { for } z \neq 0 ; \\
\frac{8}{3} l \pi R_{i}^{2} e^{-l\left(\xi-\xi^{\prime}\right) \cdot \mathbf{X}^{(i)}} & \text { for }=0 ;\end{cases} \\
& \widehat{H}_{22}^{(i)}= \begin{cases}-\frac{4 \imath \pi R_{i}^{2}\left[2 z c s_{1}^{2} s_{2}^{2}-c_{1}^{2} s_{2}^{2}(z c-s)-z^{2} s-2 s_{1}^{2} s_{2}^{2} s+z^{2} s_{1}^{2} s_{2}^{2} s+c_{2}^{2}(s-z c)\right]}{z^{3}} e^{-l\left(\xi-\xi^{\prime}\right) \cdot \mathbf{X}^{(i)}} & \text { for } z \neq 0 ; \\
\frac{8}{3} l \pi R_{i}^{2} e^{-l\left(\xi-\xi^{\prime}\right) \cdot \mathbf{X}^{(i)}} & \text { for } z=0 ;\end{cases} \\
& \widehat{H}_{33}^{(i)}= \begin{cases}-\frac{4 \imath \pi R_{i}^{2}\left[-z c s_{1}^{2}+\left(s_{1}^{2}-z^{2}\right) s+2 z c c_{1}^{2}+c_{1}^{2} s\left(z^{2}-2\right)\right]}{z^{3}} e^{-l\left(\xi-\xi^{\prime}\right) \cdot \mathbf{X}^{(i)}} & \text { for } z \neq 0 ; \\
\frac{8}{3} l \pi R_{i}^{2} e^{-l\left(\xi-\xi^{\prime}\right) \cdot \mathbf{X}^{(i)}} & \text { for } z=0 ;\end{cases}
\end{aligned}
$$




$$
\begin{aligned}
& \widehat{H}_{12}^{(i)}=\widehat{H}_{21}^{(i)} \\
& = \begin{cases}-\frac{4 l \pi R_{i}^{2} s_{1}^{2} c_{2} s_{2}\left[3 z c+\left(z^{2}-3\right) s\right]}{z^{3}} e^{-\imath\left(\xi-\xi^{\xi}\right) \cdot \mathbf{X}^{(i)}} & \text { for } z \neq 0 ; \\
0 & \text { for } z=0\end{cases} \\
& \widehat{H}_{13}^{(i)}=\widehat{H}_{31}^{(i)} \\
& = \begin{cases}-\frac{4 l \pi R_{i}^{2} c_{2} c_{1} s_{1}\left[3 z c+\left(z^{2}-3\right) s\right]}{z^{3}} e^{-l\left(\xi-\xi^{\prime}\right) \cdot \mathbf{X}^{(i)}} & \text { for } z \neq 0 ; \\
0 & \text { for } z=0 ;\end{cases} \\
& \widehat{H}_{23}^{(i)}=\widehat{H}_{32}^{(i)} \\
& = \begin{cases}-\frac{4 \iota \pi R_{i}^{2} c_{1} s_{1} s_{2}\left[3 z c+\left(z^{2}-3\right) s\right]}{z^{3}} e^{-l\left(\xi-\xi^{\prime}\right) \cdot \mathbf{X}^{(i)}} & \text { for } z \neq 0 ; \\
0 & \text { for } z=0\end{cases} \\
& \widehat{P}_{1}^{(i)}= \begin{cases}\frac{4 \imath \pi R_{i} c_{2} s_{1}(z c-s)}{z^{2}} e^{-l\left(\xi-\xi^{\prime}\right) \cdot \mathbf{X}^{(i)}} & \text { for } z \neq 0 \\
0 & \text { for } z=0 ;\end{cases} \\
& \widehat{P}_{2}^{(i)}= \begin{cases}\frac{4 l \pi R_{i} s_{1} s_{2}(z c-s)}{z^{2}} e^{-\imath\left(\xi-\xi^{\prime}\right) \cdot \mathbf{X}^{(i)}} & \text { for } z \neq 0 ; \\
0 & \text { for } z=0 ;\end{cases} \\
& \widehat{P}_{3}^{(i)}= \begin{cases}\frac{4 \iota \pi R_{i} c_{1}(z c-s)}{z^{2}} e^{-\iota\left(\xi-\xi^{\prime}\right) \cdot \mathbf{X}^{(i)}} & \text { for } z \neq 0 ; \\
0 & \text { for } z=0 ;\end{cases}
\end{aligned}
$$

where $c=\cos (z), s=\sin (z), s_{1}=\sin \theta_{0} ; c_{1}=\cos \theta_{0} ; s_{2}=\sin \varphi_{0}$ and $c_{2}=\cos \varphi_{0}$ with $\theta_{0}$ and $\varphi_{0}$ being the elevation and azimuthal angles of the discrete vector $\xi-\xi^{\prime}$.

$D$. The expressions for the components $\widehat{Q}_{m j}^{(i)}\left(\xi-\xi^{\prime}\right)$ of matrix $\widehat{\mathbf{Q}}^{(i)}\left(\boldsymbol{\xi}-\boldsymbol{\xi}^{\prime}\right)$ in Eq. (43)

(i) when the composite with circular $i$ th-inclusion of radius $R_{i}$ is under consideration

$$
\begin{aligned}
& \widehat{Q}_{11}^{(i)}= \begin{cases}2 \pi R_{i}\left[\frac{J_{1}(z)}{z}-c^{2} J_{2}(z)\right] e^{-\imath\left(\xi-\xi^{\prime}\right) \cdot \mathbf{X}^{(i)}} & \text { for } z \neq 0 ; \\
\pi R_{i} e^{-l\left(\xi-\xi^{\prime}\right) \cdot \mathbf{X}^{(i)}} & \text { for } z=0 ;\end{cases} \\
& \widehat{Q}_{22}^{(i)}= \begin{cases}2 \pi R_{i}\left[\frac{J_{1}(z)}{z}-s^{2} J_{2}(z)\right] e^{-\imath\left(\xi-\xi^{\prime}\right) \cdot \mathbf{X}^{(i)}} & \text { for } z \neq 0 ; \\
\pi R_{i} e^{-l\left(\xi-\xi^{\prime}\right) \cdot \mathbf{X}^{(i)}} & \text { for } z=0 ;\end{cases} \\
& \widehat{Q}_{12}^{(i)}=\widehat{Q}_{21}^{(i)}= \begin{cases}-2 \pi R_{i} c s J_{2}(z) e^{-l\left(\xi-\xi^{\prime}\right) \cdot \mathbf{X}^{(i)}} & \text { for } z \neq 0 ; \\
0 & \text { for } z=0 ;\end{cases}
\end{aligned}
$$

(ii) when the composite with spherical $i$ th-inclusion of radius $R_{i}$ is concerned

$$
\begin{aligned}
& \widehat{Q}_{33}^{(i)}= \\
& \begin{cases}\frac{4 \pi R_{i}^{2}\left[s_{1}^{2}(s-z c)+2 z c c_{1}^{2}+c_{1}^{2} s\left(z^{2}-2\right)\right]}{z^{3}} e^{-l\left(\xi-\xi^{\prime}\right) \cdot \mathbf{X}^{(i)}} & \text { for } z \neq 0 ; \\
\frac{4}{3} \pi R_{i}^{2} e^{-l\left(\xi-\xi^{\prime}\right) \cdot \mathbf{X}^{(i)}} & \text { for } z=0 ;\end{cases}
\end{aligned}
$$

$$
\begin{aligned}
\hat{Q}_{12}^{(i)} & =\hat{Q}_{21}^{(i)} \\
& = \begin{cases}\frac{4 \pi R_{i}^{2} s_{1}^{2} c_{2} s_{2}\left[3 z c+\left(z^{2}-3\right) s\right]}{z^{3}} e^{-l\left(\xi-\xi^{\prime}\right) \cdot \mathbf{X}^{(i)}} & \text { for } z \neq 0 ; \\
0 & \text { for } z=0 ;\end{cases}
\end{aligned}
$$

$$
\widehat{Q}_{13}^{(i)}=\hat{Q}_{31}^{(i)}
$$$$
= \begin{cases}\frac{4 \pi R_{i}^{2} c_{2} c_{1} s_{1}\left[3 z c+\left(z^{2}-3\right) s\right]}{z^{3}} e^{-\iota\left(\xi-\xi^{\prime}\right) \cdot \mathbf{X}^{(i)}} & \text { for } z \neq 0 ; \\ 0 & \text { for } z=0 ;\end{cases}
$$$$
\widehat{Q}_{23}^{(i)}=\hat{Q}_{32}^{(i)}
$$$$
= \begin{cases}\frac{4 \pi R_{i}^{2} c_{1} s_{1} s_{2}\left[3 z c+\left(z^{2}-3\right) s\right]}{z^{3}} e^{-l\left(\xi-\xi^{\prime}\right) \cdot \mathbf{X}^{(i)}} & \text { for } z \neq 0 ; \\ 0 & \text { for } z=0 ;\end{cases}
$$

E.The expressions for the components $\widehat{L}_{m l j}^{(i)}\left(\xi-\xi^{\prime}\right)$ of matrix $\widehat{\mathbb{L}}(\xi)(\xi)$ in $E q .(43)$

(i) when the composite with circular inclusions of radius $R_{i}$ is under consideration

$$
\begin{aligned}
& \widehat{L}_{111}^{(i)}=-\widehat{L}_{212}^{(i)}=-\widehat{L}_{221}^{(i)}=-2 \pi R_{i}^{2} c\left[s^{2} J_{3}(z)-\frac{J_{2}(z)}{z}\right] e^{-l\left(\xi-\xi^{\prime}\right) \cdot \mathbf{X}^{(i)}} \\
& \widehat{L}_{121}^{(i)}=\widehat{L}_{112}^{(i)}=-\widehat{L}_{222}^{(i)}=-2 \pi R_{i}^{2} S\left[-c^{2} J_{3}(z)+\frac{J_{2}(z)}{z}\right] e^{-\iota\left(\xi-\xi^{\prime}\right) \cdot \mathbf{x}^{(i)}} \\
& \widehat{L}_{122}^{(i)}=-2 \pi R_{i}^{2} c\left[c^{2} J_{3}(z)-\frac{3 J_{2}(z)}{z}\right] e^{-\iota\left(\xi-\xi^{\prime}\right) \cdot \mathbf{x}^{(i)}} \\
& \widehat{L}_{211}^{(i)}=-2 \pi R_{i}^{2} s\left[s^{2} J_{3}(z)-\frac{3 J_{2}(z)}{z}\right] e^{-\iota\left(\xi-\xi^{\prime}\right) \cdot \mathbf{x}^{(i)}} \\
& \text { for } z \neq 0 \text { and } \widehat{L}_{m l j}^{(i)}=0 \text { for } z=0
\end{aligned}
$$

$$
\begin{aligned}
& \hat{Q}_{11}^{(i)}= \begin{cases}\frac{4 \pi R_{i}^{2}\left[(s-z c)\left(s_{2}^{2}+c_{1}^{2} c_{2}^{2}\right)+2 z c c_{2}^{2} s_{1}^{2}+c_{2}^{2} s_{1}^{2} s\left(z^{2}-2\right)\right]}{z^{3}} e^{-l\left(\xi-\xi^{\prime}\right) \cdot \mathbf{X}^{(i)}} & \text { for } z \neq 0 ; \\
\frac{4}{3} \pi R_{i}^{2} e^{-l\left(\xi-\xi^{\prime}\right) \cdot \mathbf{X}^{(i)}} & \text { for } z=0 ;\end{cases} \\
& \widehat{Q}_{22}^{(i)}= \begin{cases}\frac{4 \pi R_{i}^{2}\left[c_{2}^{2}(s-z c)+s_{2}^{2} c_{1}^{2}(s-z c)+2 z c s_{2}^{2} s_{1}^{2}+s_{2}^{2} s_{1}^{2} s\left(z^{2}-2\right)\right]}{z^{3}} e^{-l\left(\xi-\xi^{\prime}\right) \cdot \mathbf{x}^{(i)}} & \text { for } z \neq 0 ; \\
\frac{4}{3} \pi R_{i}^{2} e^{-l\left(\xi-\xi^{\prime}\right) \cdot \mathbf{X}^{(i)}} & \text { for } z=0 ;\end{cases}
\end{aligned}
$$


(ii) when the composite with spherical inclusions of radius $R i$ is concerned

$$
\begin{aligned}
& \widehat{L}_{111}^{(i)}=4 \pi R_{i}^{3} z^{-4} c_{2} s_{1}\left\{\begin{array}{l}
z c\left[-z^{2}+9 c_{1}^{2} c_{2}^{2}+\left(z^{2}-6\right) c_{2}^{2} s_{1}^{2}+9 s_{2}^{2}\right] \\
+\left[z^{2}+3\left(z^{2}-3\right) c_{1}^{2} c_{2}^{2}-3\left(z^{2}-2\right) c_{2}^{2} s_{1}^{2}-9 s_{2}^{2}+3 z^{2} s_{2}^{2}\right] s
\end{array}\right\} e-\iota\left(\boldsymbol{\xi}-\boldsymbol{\xi}^{\prime}\right) \cdot \mathbf{X}^{(i)} \\
& \widehat{L}_{222}^{(i)}=4 \pi R_{i}^{3} z^{-4} s_{1} s_{2}\left\{\begin{array}{l}
z c\left(9 c_{2}^{2}-z^{2}+9 c_{1}^{2} s_{2}^{2}-6 s_{1}^{2} s_{2}^{2}+z^{2} s_{1}^{2} s_{2}^{2}\right) \\
+\left[z^{2}+3\left(z^{2}-3\right) c_{2}^{2}+3\left(z^{2}-3\right) c_{1}^{2} s_{2}^{2}+6 s_{1}^{2} s_{2}^{2}-3 z^{2} s_{1}^{2} s_{2}^{2}\right] s
\end{array}\right\} e^{-\iota\left(\xi-\xi^{\prime}\right) \cdot \mathbf{X}^{(i)}} \\
& \widehat{L}_{333}^{(i)}=2 \pi R_{i}^{3} z^{-4} c_{1}\left\{\begin{array}{c}
z c\left[3-z^{2}+\left(z^{2}-15\right)\left(2 c_{1}^{2}-1\right)\right] \\
+\left[8 z^{2}-18+\left(30-12 z^{2}\right) c_{1}^{2}\right] s
\end{array}\right\} e^{-i\left(\xi-\xi^{\prime}\right) \cdot \mathbf{X}^{(i)}} \\
& \widehat{L}_{122}^{(i)}=4 \pi R^{3} z^{-4} c_{2} s_{1}\left\{\begin{array}{l}
z c\left[-z^{2}+3 c_{2}^{2}+\left(3 c_{1}^{2}+\left(z^{2}-12\right) s_{1}^{2}\right) s_{2}^{2}\right] \\
+\left[z^{2}+\left(z^{2}-3\right) c_{2}^{2}+\frac{1}{2}\left(9-4 z^{2}+3\left(2 z^{2}-5\right)\left(2 c_{1}^{2}-1\right)\right) s_{2}^{2}\right] s
\end{array}\right\} e^{-l\left(\xi-\xi^{\prime}\right) \cdot \mathbf{X}^{(i)}} \\
& \widehat{L}_{133}^{(i)}=2 \pi R_{i}^{3} z^{-4} c_{2} s_{1}\left\{\begin{array}{l}
z\left[-9-z^{2}+\left(z^{2}-15\right)\left(2 c_{1}^{2}-1\right)\right] c \\
+\left[9-2 z^{2}+\left(15-6 z^{2}\right)\left(2 c_{1}^{2}-1\right)\right] s
\end{array}\right\} e^{-\imath\left(\xi-\xi^{\prime}\right) \cdot \mathbf{X}^{(i)}} \\
& \widehat{L}_{211}^{(i)}=2 \pi R_{i}^{3} z^{-4} s_{1} s_{2}\left\{\begin{array}{l}
2 z c\left[c_{2}^{2}\left(3 c_{1}^{2}+\left(z^{2}-12\right) s_{1}^{2}\right)+3 s_{2}^{2}-z^{2}\right] \\
+\left[\left(9-4 z^{2}+3\left(2 z^{2}-5\right)\left(2 c_{1}^{2}-1\right)\right) c_{2}^{2}+2 z^{2}+\left(z^{2}-3\right) s_{2}^{2}\right] s
\end{array}\right\} e^{-l\left(\xi-\xi^{\prime}\right) \cdot \mathbf{X}^{(i)}} \\
& \widehat{L}_{233}^{(i)}=2 \pi R_{i}^{3} z^{-4} s_{1} s_{2}\left\{\begin{array}{l}
z\left[-9-z^{2}+\left(z^{2}-15\right)\left(2 c_{1}^{2}-1\right)\right] c \\
+\left[9-2 z^{2}+\left(15-6 z^{2}\right)\left(2 c_{1}^{2}-1\right)\right] s
\end{array}\right\} e^{-\iota\left(\xi-\xi^{\prime}\right) \cdot \mathbf{X}^{(i)}} \\
& \widehat{L}_{311}^{(i)}=4 \pi R_{i}^{3} z^{-4} c_{1}\left\{\begin{array}{l}
z c\left[3 c_{1}^{2} c_{2}^{2}-z^{2}+\left(z^{2}-12\right) c_{2}^{2} s_{1}^{2}+3 s_{2}^{2}\right] \\
+\left[z^{2}+\left(z^{2}-3\right) c_{1}^{2} c_{2}^{2}-\left(5 z^{2}-15\right) c_{2}^{2} s_{1}^{2}-3 s_{2}^{2}+z^{2} s_{2}^{2}\right] s
\end{array}\right\} e^{-c\left(\xi-\xi^{\prime}\right) \cdot \mathbf{x}^{(i)}} \\
& \widehat{L}_{322}^{(i)}=4 \pi R_{i}^{3} z^{-4} c_{1}\left\{\begin{array}{l}
z c\left[-z^{2}+3 c_{2}^{2}+3 c_{1}^{2} s_{2}^{2}-12 s_{1}^{2} s_{2}^{2}+z^{2} s_{1}^{2} s_{2}^{2}\right] \\
+\left[z^{2}+\left(z^{2}-3\right) c_{2}^{2}+\left(z^{2}-3\right) c_{1}^{2} s_{2}^{2}+12 s_{1}^{2} s_{2}^{2}-5 z^{2} s_{1}^{2} s_{2}^{2}\right] s
\end{array}\right\} e^{-l\left(\xi-\xi^{\prime}\right) \cdot \mathbf{x}^{(i)}} \\
& \widehat{L}_{112}^{(i)}=\widehat{L}_{121}^{(i)}=2 \pi R_{i}^{3} z^{-4} s_{1} s_{2}\left\{\begin{array}{c}
2 s_{2}^{2}\left[3 z c+\left(z^{2}-3\right) s\right] \\
-z c_{2}^{2} c\left[9-z^{2}+\left(z^{2}-15\right)\left(2 c_{1}^{2}-1\right)\right] \\
+\left[9-4 z^{2}+3\left(2 z^{2}-5\right)\left(2 c_{1}^{2}-1\right)\right] s c_{2}^{2}
\end{array}\right\} e^{-l\left(\xi-\xi^{\prime}\right) \cdot \mathbf{X}^{(i)}} \\
& \widehat{L}_{113}^{(i)}=\widehat{L}_{131}^{(i)}=4 \pi R_{i}^{3} z^{-4} c_{1}\left\{\begin{array}{c}
c_{2}^{2} s_{1}^{2}\left[z\left(z^{2}-12\right) c+\left(12-5 z^{2}\right) s\right] \\
+\left(c_{1}^{2} c_{2}^{2}+s_{2}^{2} s\right)\left[3 z c+\left(z^{2}-3\right) s\right]
\end{array}\right\} e^{-\iota\left(\xi-\xi^{\prime}\right) \cdot \mathbf{x}^{(i)}} \\
& \widehat{L}_{221}^{(i)}=\widehat{L}_{212}^{(i)}=2 \pi R_{i}^{3} z^{-4} c_{2} s_{1}\left\{\begin{array}{c}
2 c_{2}^{2}\left[3 z c+\left(z^{2}-3\right) s\right] \\
-s_{2}^{2} z c\left[9-z^{2}+\left(z^{2}-15\right)\left(2 c_{1}^{2}-1\right)\right] \\
+\left[9-4 z^{2}+3\left(2 z^{2}-5\right)\left(2 c_{1}^{2}-1\right)\right] s_{2}^{2} s
\end{array}\right\} e^{-i\left(\xi-\xi^{\prime}\right) \cdot \mathbf{X}^{(i)}} \\
& \hat{L}_{223}^{(i)}=\hat{L}_{232}^{(i)}=2 \pi R_{i}^{3} z^{-4} c_{1}\left\{\begin{array}{c}
c_{2}^{2}\left[3 z c+\left(z^{2}-3\right) s\right] \\
+s_{2}^{2} s_{1}^{2}\left[z\left(z^{2}-12\right) c+\left(12-5 z^{2}\right) s\right] \\
+s_{2}^{2} c_{1}^{2}\left[3 z c+\left(z^{2}-3\right) s\right]
\end{array}\right\} e^{-l\left(\xi-\xi^{\prime}\right) \cdot \mathbf{X}^{(i)}} \\
& \widehat{L}_{331}^{(i)}=\widehat{L}_{313}^{(i)}=4 \pi R_{i}^{3} z^{-4} c_{2} s_{1}\left\{\begin{array}{c}
c_{1}^{2}\left[z\left(z^{2}-12\right) c+\left(12-5 z^{2}\right) s\right] \\
+s_{1}^{2}\left[3 z c+\left(z^{2}-3\right) s\right]
\end{array}\right\} e^{-l\left(\xi-\xi^{\prime}\right) \cdot \mathbf{x}^{(i)}} \\
& \widehat{L}_{332}^{(i)}=\widehat{L}_{323}^{(i)}=4 \pi R_{i}^{3} z^{-4} s_{1} s_{2}\left\{\begin{array}{c}
c_{1}^{2}\left[z\left(z^{2}-12\right) c+\left(12-5 z^{2}\right) s\right] \\
+s_{1}^{2}\left[3 z c+\left(z^{2}-3\right) s\right]
\end{array}\right\} e^{-l\left(\xi-\xi^{\prime}\right) \cdot \mathbf{x}^{(i)}}
\end{aligned}
$$




$$
\begin{aligned}
& \begin{aligned}
\widehat{L}_{123}^{(i)} & =\widehat{L}_{132}^{(i)}=\widehat{L}_{231}^{(i)}=\widehat{L}_{213}^{(i)}=\widehat{L}_{312}^{(i)}=\widehat{L}_{321}^{(i)} \\
& =4 \pi R_{i}^{3} z^{-4} c_{1} s_{1}^{2} c_{2} s_{2}\left[z\left(z^{2}-15\right) c+3\left(5-2 z^{2}\right) s\right] e^{-l\left(\xi-\xi^{\prime}\right) \cdot \mathbf{X}^{(i)}}
\end{aligned} \\
& \text { for } z \neq 0 \text { and } \widehat{L}_{m l j}^{(i)}=0 \text { for } z=0 .
\end{aligned}
$$

\section{References}

[1] Y. Benveniste, A general interface model for a three-dimensional curved thin anisotropic interphase between two anisotropic media, J. Mech. Phys. Solids 54 (2006) 708-734.

[2] D.J. Bottomley, T. Ogino, Alternative to the Shuttleworth formulation of solid surface stress, Phys. Rev. B63 (2001) 165412-1-165412-5.

3] Bonnet, Effective properties of elastic periodic composite media with fibres, J. Mech. Phys. Solids 55 (2007) 881-899.

[4] P. Bövik, On the modelling of thin interface layers in elastic and acoustic scattering problems, Q. J. Mech. Appl. Math. 47 (1994) $17-42$

[5] R. Brenner, Numerical computation of the response of piezoelectric composites using Fourier transform, Phys. Rev. B79 (2009) 184106-184111.

[6] O.P. Bruno, The effective conductivity of strongly heteroheneous composites, Proc. R Soc. Lond A433 (1991) 353-381.

7] J.W. Cahn, Surface stress and the chemical equilibrium of small crystals - I. The case of the isotropic surface, Acta Metall. 28 (1980) 1333-1338.

[8] T. Chen, M.-S. Chiu, C.-N. Weng, Derivation of the generalized Young-Laplace equation of curved interfaces in nanoscaled solids, J. Appl. Phys. 100 (2006) 074308-1-074308-5.

[9] H. Cheng, S. Torquato, Effective conductivity of dispersion of spheres with a superconducting interface, Proc. R. Soc. Lond. A453 (1997) 1331-1344.

[10] R.M. Christensen, K.H. Lo, Solutions for effective shear properties in three phase sphere and cylinder models, J. Mech. Phys. Solids 27 (1979) 315-330.

[11] R. Dingreville, J. Qu, M. Cherkaoui, Surface free energy and its effect on elastic behavior of nano-sized particles, wires and films, J. Mech. Phys. Solids 8 (2005) $1827-1854$

[12] M.E. Gurtin, A.I. Murdoch, A continuum theory of elastic material surfaces, Arch. Ration. Mech. Anal. 57 (1975) 291-323.

13] Z. Hashin, Theory of Fiber Reinforced Materials NASA Contract Report No. CR 1974 (1972) pp. 67-383.

[14] Z. Hashin, Thin interphase/imperfect interface in conduction, J. Appl. Phys. 84 (2001) $2261-2267$

15] E. Hervé, Thermal and thermoelastic behaviour of multiply coated inclusion-reinforced composites, Int. J. Solids Struct. 39 (2002) 1041-1058.

[16] R. Hill, Discontinuity relations in mechanics of solids. in: I.N. Sneddon, R. Hill (Eds.), Progress in Solid Mechanics, vol. 2. North-Holland, Amsterdam, 1999, pp. 247-276.
[17] P.L. Kapitza, The study of heat transfer in helium II, J. Phys. (USSR) 4 (1941)

[18] H. Le Quang, G. Bonnet Q-C. He, Effective thermal conductivity of inhomogeneous materials with highly conducting imperfect interfaces, Phys. Rev. B81 (2010) 064203.

19] R. Lipton, Reciprocal relations, bounds, and size effects for composites with highly conducting interface, SIAM J. Appl. Math. 57 (1997) 347-363.

20] R. Lipton, Variational methods, bounds, and size effects for composites with highly conducting interface, J. Mech. Phys. Solids 45 (1997) 361-384.

[21] R. Lipton, B. Vernescu, Composites with imperfect interface, Proc. Phys. Soc (1996) 329-358.

22] R. Lipton, D.R.S. Talbot, Bounds for the effective conductivity of a composite with an imperfect interface, Proc. Phys. Soc. A457 (2001) 1501-1517.

[23] J. Michel, H. Moulinec, P. Suquet, Effective properties of composite materials with periodic microstructure: a computational approach, Comput. Methods Appl. Mech. Eng. 172 (1999) 109-143.

24] R.E. Miller, V.B. Shenoy, Size-dependent elastic properties of nanosized structural elements, Nanotechnology 11 (2000) 139-147.

25] T. Miloh, Y. Benveniste, On the effective conductivity of composites with ellipsoidal inhomogeneities and highly conducting interfaces, Proc. R. Soc. Lond. 455 (1999) 2687-2706

26] G.W. Milton, The Theory of Composites. Cambridge University Press, London, 2002 .

[27] H. Moulinec, P. Suquet, A fast numerical method for computing the linear and nonlinear properties of composites, C. R. Acad. Sci. II 318 (1994) 1417-1423.

28] H. Pham Huy, E. Sanchez-Palencia, Phénomènes de transmission à travers des couches minces de conductivité élevée, J. Math. Anal. Appl. 47 (1974) 284-309.

29] Y.Z. Povstenko, Theoretical investigation of phenomena caused by heterogeneous surface tension in solids, J. Mech. Phys. Solids 41 (1993) $1499-1514$.

[30] E. Sanchez-Palencia, Comportement limite d'un problème de transmission travers une plaque faiblement conductrice, C. R. Acad. Sci. Paris Ser. A270 (1970) $1026-1028$

[31] R. Shuttleworth, The surface tension of solid, Proc. Phys. Soc. A63 (1950) 444-457.

[32] J.C. Smith, Correction and extension of van der Poel's method for calculating the shear modulus of a particulate composite, J. Res. Natl. Bur. Stand. 78A (1974) 355-361.

[33] J.C. Smith, Simplification of van der Poel's formula for the shear modulus of a particulate composite, J. Res. Nat. Bur. Stand. 79A (1975) 419-423.

[34] C. Van der Poel, On the rheology of concentrated suspensions, Rheol. Acta (1958) $198-205$

[35] J. Yvonnet, Q.-C. He, C. Toulemonde, Numerical modelling of the effective conductivities of composites with arbitrarily shaped inclusions and highly conducting interface, Compos. Sci. Technol. 68 (2008) 2818-2825. 